\title{
Deciphering the Active Compounds and Mechanisms of Qixuehe Capsule on Qi Stagnation and Blood Stasis Syndrome: A Network Pharmacology Study
}

\author{
Yu-Xi Huang $\left(\mathbb{D},{ }^{1,2}\right.$ Ding-Qiao Xu, ${ }^{1}$ Shi-Jun Yue ${ }^{D},{ }^{1}$ Yan-Yan Chen ${ }^{D},{ }^{1}$ Hui-Juan Tao, ${ }^{3}$ \\ Rui-jia Fu, ${ }^{1}$ Li-Ming Xing, ${ }^{1}$ Taiyi Wang, ${ }^{2}$ Yu-ling Ma, ${ }^{2}$ Bao-An Wang, ${ }^{4}$ Yu-Ping Tang $\mathbb{D}^{1,3}$ \\ and Jin-Ao Duan ${ }^{3}$ \\ ${ }^{1}$ Key Laboratory of Shaanxi Administration of Traditional Chinese Medicine for TCM Compatibility, \\ State Key Laboratory of Research \& Development of Characteristic Qin Medicine Resources (Cultivation), \\ Shaanxi Key Laboratory of Chinese Medicine Fundamentals and New Drugs Research, \\ Shaanxi Collaborative Innovation Center of Chinese Medicinal Resources Industrialization, \\ Shaanxi University of Chinese Medicine, Xi'an 712046, China \\ ${ }^{2}$ Oxford Chinese Medicine Research Centre, University of Oxford, Oxford, UK \\ ${ }^{3}$ Jiangsu Collaborative Innovation Center of Chinese Medicinal Resources Industrialization, \\ Jiangsu Key Laboratory for High Technology Research of TCM Formulae, \\ National and Local Collaborative Engineering Center of Chinese Medicinal Resources Industrialization and Formulae \\ Innovative Medicine, Nanjing University of Chinese Medicine, Nanjing 210023, China \\ ${ }^{4}$ Shaanxi Momentum Qixuehe Pharmaceutical Co., Ltd., Xi'an 712000, China
}

Correspondence should be addressed to Shi-Jun Yue; shijun_yue@163.com and Yu-Ping Tang; yupingtang@sntcm.edu.cn

Received 14 October 2019; Revised 18 January 2020; Accepted 25 January 2020; Published 27 February 2020

Academic Editor: Michel M. Machado

Copyright (C) $2020 \mathrm{Yu}$-Xi Huang et al. This is an open access article distributed under the Creative Commons Attribution License, which permits unrestricted use, distribution, and reproduction in any medium, provided the original work is properly cited.

Background. Qixuehe capsule (QXH), a Chinese patent medicine, has been demonstrated to be effective in the treatment of menstrual disorders. In traditional Chinese medicine (TCM) theory, qi stagnation and blood stasis syndrome (QS-BSS) is the main syndrome type of menstrual disorders. However, the pharmacodynamic effect of QXH in treating QS-BSS is not clear, and the main active compounds and underlying mechanisms remain unknown. Methods. A rat model of QS-BSS was established to evaluate the pharmacodynamic effect of QXH. Thereafter, a network pharmacology approach was performed to decipher the active compounds and underlying mechanisms of QXH. Results. QXH could significantly reduce the rising whole blood viscosity (WBV) and plasma viscosity (PV) but also normalize prothrombin time (PT), activated partial thromboplastin time (APTT), thrombin time (TT), and fibrinogen (FIB) content in QS-BSS rats. Based on partial least-squares-discriminant analysis (PLS-DA), the low-dose QXH-intervened (QXH-L) and the high-dose QXH-intervened (QXH-H) groups seemed the most effective by calculating the relative distance to normality. Through network pharmacology, QXH may improve hemorheological abnormality mainly via 185 compounds- 51 targets- 28 pathways, whereas 184 compounds- 68 targets- 28 pathways were associated with QXH in improving coagulopathy. Subsequently, 25 active compounds of QXH were verified by UPLC-Q/TOF-MS. Furthermore, 174 active compounds of QXH were shared in improving hemorheological abnormality and coagulopathy in QS-BSS, each of which can act on multiple targets to be mainly involved in complement and coagulation cascades, leukocyte transendothelial migration, PPAR signaling pathway, VEGF signaling pathway, and arachidonic acid metabolism. The attribution of active compounds indicated that Angelicae Sinensis Radix (DG), Paeoniae Radix Rubra (CS), Carthami Flos (HH), Persicae Semen (TR), and Corydalis Rhizoma (YHS) were the vital herbs of QXH in treating QS-BSS. Conclusion. QXH can improve the hemorheology abnormality and coagulopathy of QS-BSS, which may result from the synergy of multiple compounds, targets, and pathways. 


\section{Introduction}

Qixuehe capsule $(\mathrm{QXH})$ is a Chinese patent medicine and used clinically for the treatment of menstrual disorders for a dozen years. Menstrual disorders are known to be a common gynecological disease, which is characterized by abnormal menstrual cycle, dysmenorrhea, amenorrhea, uterine bleeding, postpartum abdominal pain, lochia, breast swelling, infertility, and chloasma, seriously affecting female's health $[1,2]$. Although the symptoms of menstrual disorders are complex and diverse, qi stagnation and blood stasis syndrome (QS-BSS) is the main syndrome type of menstrual disorders in traditional Chinese medicine (TCM) theory. QS-BSS is defined as due to the poor stagnation of the operation of the qi, the blood is running impediment and the pathological state of blood stasis occurs, which is mainly related to menstrual disorders, hyperlipidemia, liver injury, ischemic brain injury, and hypertension [3-5]. The formation of QS-BSS is accompanied by the following pathological changes: (1) abnormal hemorheology; (2) increased blood coagulability or reduced fibrinolytic activity; (3) microcirculation disturbance; (4) increased platelet aggregation or decreased release function; (5) hemodynamic disturbance; (6) manifestations of static blood in pathological section; (7) vessel obstruction [6]. Numerous studies have shown that the pathological changes of hemorheological abnormality and coagulopathy are more important during the formation of QS-BSS [7, 8]. TCM used to treat syndrome by compound formulas, which are composed of several or even tens of herbal medicines in different quantities, following the composition theory "Monarch, Minister, Assistant and Guide" [9]. QXH is based on the addition and subtraction of Xuefu Zhuyu decoction (a classic blood-activating formula) and Chaihu Shugan powder (a classic qi-regulating formula), which consists of Angelicae Sinensis Radix (DG), Paeoniae Radix Rubra (CS), Chuanxiong Rhizoma (CX), Persicae Semen (TR), Bupleuri Radix $(\mathrm{CH})$, Carthami Flos $(\mathrm{HH})$, Salviae Miltiorrhizae Radix et Rhizoma (DS), etc., so as to regulate liver-qi stagnation, promote blood circulation, and remove blood stasis in the treatment of QS-BSS [10, 11]. However, the potential active compounds and underlying mechanisms of QXH in treating QS-BSS remain unclear.

In recent years, network pharmacology has been proposed as a promising approach for understanding compound formulas $[12,13]$. Studies have shown that Xuefu Zhuyu decoction and compound Danshen formula have been analyzed by network pharmacology, and the main active compounds in the formulas and the treatment mechanisms have been elucidated $[14,15]$. Therefore, network pharmacology is promising to decipher the active compounds and possible mechanisms of QXH.

In this study, we evaluated the pharmacodynamic effect of QXH in the treatment of QS-BSS based on animal experiments from the two aspects: hemorheology and coagulation function. Hemorheological abnormality is reflected in changes in blood fluidity, deformability, and viscosity, which can be measured by whole blood viscosity (WBV) and plasma viscosity (PV). Meanwhile, coagulopathy is reflected in the abnormality of coagulation factor function; thrombin time (TT), prothrombin time (PT), activated partial thromboplastin time (APTT), and fibrinogen content (FIB) are the important monitoring index of coagulopathy in clinical aspects [16]. Subsequently, a network pharmacology approach was performed to decipher the active compounds of QXH and its regulation mechanisms of QS-BSS. By constructing two herb-compound-pathological process relationship diagrams, the possible mechanisms of QXH in the treatment of QS-BSS were systematically elucidated. A workflow of the entire study procedure is depicted in Figure 1.

\section{Materials and Methods}

2.1. Drugs and Reagents. QXH (Lot. 161030) was supplied by Shaanxi Momentum Pharmaceutical Co., Ltd., Shaanxi, China; compound danshen dripping pills (CDDP, Lot. 180602) were purchased from Tasly Pharmaceutical Co., Ltd., Tianjin, China; and adrenaline hydrochloride injection (Adr, Lot. 20190102) was obtained from Grand Pharmaceutical Co., Ltd., Wuhan, China. Carboxymethylcellulose sodium (Lot. 20180803) was purchased from Tianli Chemical Reagent Co., Ltd., Tianjin, China; and disposable human venous blood sample collection containers $(3.2 \%$ sodium citrate anticoagulation, blood/citrate: $9: 1$, v/v, Lot. 20190407, and heparin sodium anticoagulation, Lot. 20190705) were obtained from Yuli Medical Instrument Co., Ltd., Jiangsu, China. PT (Lot. 20190214), APTT (Lot. 20190214), TT (Lot. 20190114), and FIB (Lot. 20192012) assay kits were obtained from Steellex Biotechnology Co., Ltd., Taizhou, China. MS-grade acetonitrile and methanol were purchased from Merck, Darmstadt, Germany. MSgrade formic acid was obtained from Sigma-Aldrich, Darmstadt, Germany. Water used in the experiment was purified by using a Milli-Q water purification system (Billerica, MI, USA). Reference substances were purchased from Yuanye Bio-Technology Co., Ltd., Shanghai, China. The purity of each compound was determined to be higher than $98 \%$ by HPLC.

2.2. Animal Handling. Female Sprague-Dawley rats $(200 \pm 20 \mathrm{~g})$ were provided by Dossy Experimental Animals Co., Ltd., Chengdu, China. All procedures were conducted in accordance with the Regulations of Experimental Animal Administration issued by the State Committee of Science and Technology of the People's Republic of China and approved by the Animal Ethics Committee of Shaanxi University of Chinese Medicine.

After acclimatization for one week, the rats were randomly divided into 6 groups with 8 rats in each group: control, model, low-dose QXH-intervened (QXH-L), middle-dose QXH-intervened (QXH-M), high--dose QXH-intervened $(\mathrm{QXH}-\mathrm{H})$, and $\mathrm{CDDP}$-intervened (CDDP, the positive control) groups. The models QXH-L (clinical equivalent does, $0.432 \mathrm{~g} \cdot \mathrm{kg}^{-1}$ ), QXH-M (3 times clinical equivalent does, $1.296 \mathrm{~g} \cdot \mathrm{kg}^{-1}$ ), QXH-H (6 times clinical equivalent does, $2.592 \mathrm{~g} \cdot \mathrm{kg}^{-1}$ ), and CDDP (clinical equivalent does, $0.1 \mathrm{~g} \cdot \mathrm{kg}^{-1}$ ) groups were hypodermically injected with Adr $0.9 \mathrm{mg} \cdot \mathrm{kg}^{-1} \cdot \mathrm{d}^{-1}$, 


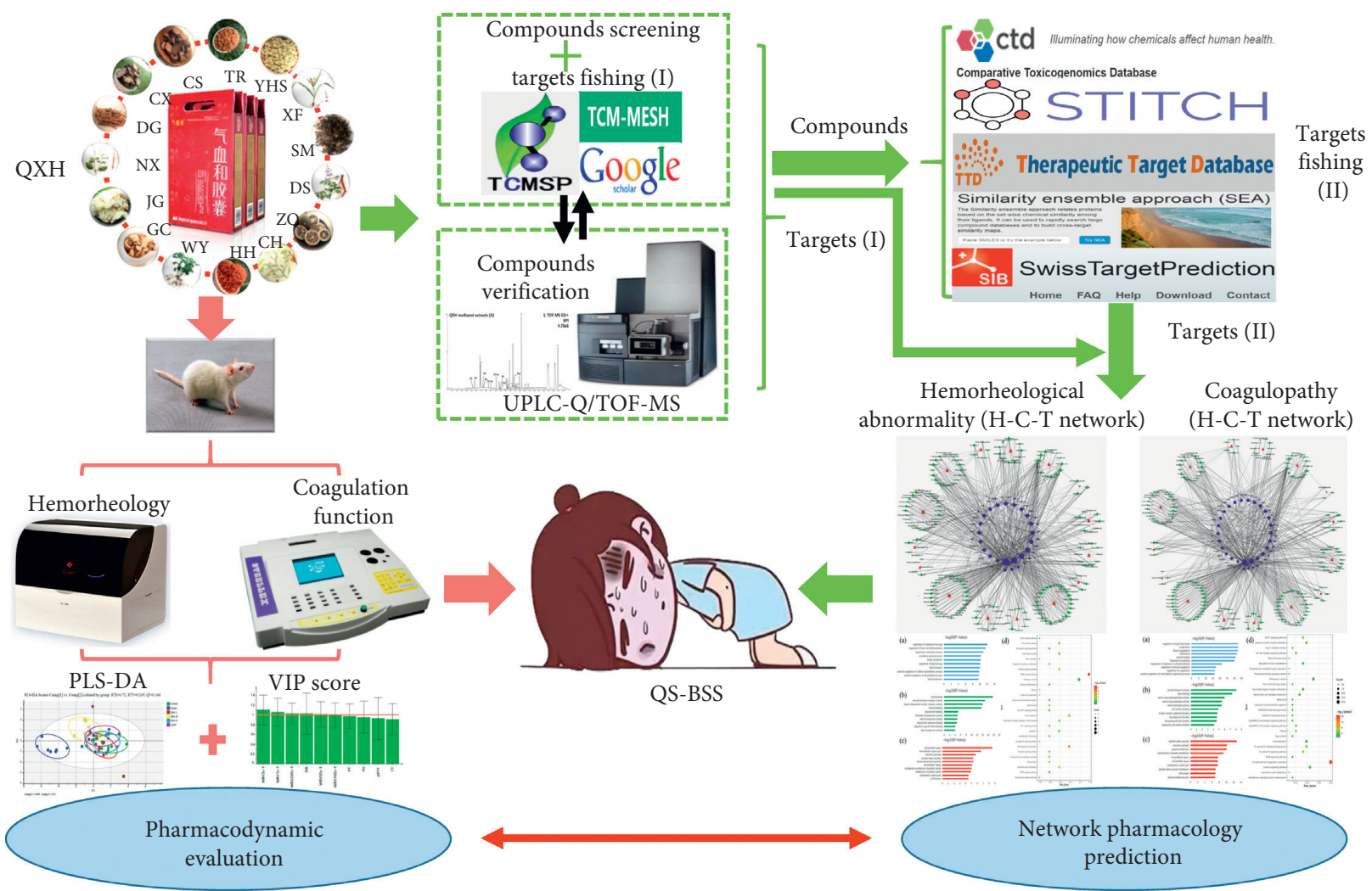

FIGURE 1: The workflow of QXH in treating QS-BSS combined with pharmacodynamic evaluation and network pharmacology prediction.

and $4 \mathrm{~h}$ later, the rats were received chronic unpredictable stimulations (A: $2 \mathrm{~h}$ ultrasound stimulation at days 1, 7, and 12; B: day and night upside down at days 2, 9, and 14; C: $2 \mathrm{~h}$ physical restraint at days 3,6 , and $11 ; \mathrm{D}: 0-2^{\circ} \mathrm{C}$ ice swim for $4 \mathrm{~min}$ at days 4, 8, and 10; E: $10 \mathrm{~min}$ tail suspension at days 5 and 10) to establish the QS-BSS rat model within 14 days. The drug dose conversion formula was as follows: human dose of crude herbs in clinic $\times 0.018 / 200 \times 1000 \times$ the multiple of clinical equivalent dose [17]. QXH and CDDP samples were dissolved in $0.5 \%$ carboxymethylcellulose sodium solution for intragastric administration. Based on the results of preexperiment, the rats in $\mathrm{QXH}$ and CDDP treatment groups were intragastrically given the corresponding dose of $\mathrm{QXH}$ and CDDP daily from the $7^{\text {th }}$ day, whereas the rats in the control and model groups were intragastrically given the same volume of $0.5 \%$ carboxymethylcellulose sodium solution [18].

2.3. Sample Collection and Indicator Measurements. On the $14^{\text {th }}$ day, after subcutaneous injection of Adr, the rats were managed to be fasting but had free access to clean water. The next morning at $8: 00$, rats were anesthetized with $10 \%$ chloral hydrate $(350 \mathrm{mg} / \mathrm{kg}$ i.p.). Thereafter, $4 \mathrm{~mL}$ blood samples of rats were collected by the abdominal aorta intubation approach into a $5 \mathrm{~mL}$ disposable human venous blood sample collection container (heparin sodium anticoagulation). Then, $800 \mu \mathrm{L}$ of whole blood was used to measure WBV at the shear rate of $1 \mathrm{~s}^{-1}, 5 \mathrm{~s}^{-1}, 50 \mathrm{~s}^{-1}$, $100 \mathrm{~s}^{-1}$, and $200 \mathrm{~s}^{-1}$, and $800 \mu \mathrm{L}$ plasma was separated from the rest of whole blood by centrifugation (3H16RI refrigerated centrifuge, Herexi Instrument \& Equipment Co., Ltd., Hunan, China) at $3000 \mathrm{rpm}$ for $10 \mathrm{~min}$ to detect PV by using a SA-5600 automatic hemorheology analyzer (Succeeder Technology Co., Ltd., Beijing, China). Meanwhile, blood samples were collected by the abdominal aorta intubation approach into a $2 \mathrm{~mL}$ disposable human venous blood sample collection container (3.2\% sodium citrate anticoagulation, blood/citrate: $9: 1, \mathrm{v} / \mathrm{v}$ ), and centrifuged at $3000 \mathrm{rpm}$ for $10 \mathrm{~min}$ immediately. $500 \mu \mathrm{L}$ plasma was used to test coagulation function (PT/s, APTT/ s, TT/s and FIB/g/L) by using a SC40 semiautomatic coagulation analyzer (Steellex Biotechnology Co., Ltd., Taizhou, China).

2.4. QXH Database Construction. The chemical compounds of 15 herbs of QXH were data-mined from relevant databases including Traditional Chinese Medicine Systems Pharmacology Database and Analysis Platform (TCMSP, http://lsp.nwu.edu.cn/index.php) [19] and TCM-MESH (http://mesh.tcm.microbioinformatics.org), and all candidate compounds were also related to the literature to determine the attribution relationship with herbs. Latin names, the number of active compounds of each herb in QXH involving in mediating hemorheological abnormality and coagulopathy, and the corresponding abbreviations of each herb are displayed in Table 1.

2.5. Active Compound Screening. Generally, a TCM formula covers various compounds, and bioactive compounds can 
TABLE 1: Latin names, the number of active compounds of each herb in QXH involving in mediating hemorheological abnormality and coagulopathy, and the corresponding abbreviations of each herb.

\begin{tabular}{|c|c|c|c|c|c|}
\hline \multirow{2}{*}{ No. } & \multirow{2}{*}{ Name } & \multirow{2}{*}{ Active compounds } & \multicolumn{2}{|l|}{ Number } & \multirow{2}{*}{ Abbreviations } \\
\hline & & & Hemorheological abnormality & Coagulopathy & \\
\hline 1 & Angelicae Sinensis Radix & 19 & 18 & 17 & DG \\
\hline 2 & Paeoniae Radix Rubra & 17 & 10 & 15 & CS \\
\hline 3 & Chuanxiong Rhizoma & 15 & 15 & 15 & $\mathrm{CX}$ \\
\hline 4 & Persicae Semen & 12 & 11 & 10 & TR \\
\hline 5 & Carthami Flos & 15 & 13 & 15 & $\mathrm{HH}$ \\
\hline 6 & Bupleuri Radix & 11 & 7 & 6 & $\mathrm{CH}$ \\
\hline 7 & Cyperi Rhizoma & 11 & 6 & 5 & $\mathrm{XF}$ \\
\hline 8 & Salviae Miltiorrhizae Radix et Rhizoma & 29 & 28 & 27 & DS \\
\hline 9 & Corydalis Rhizoma & 33 & 30 & 30 & YHS \\
\hline 10 & Platycodonis Radix & 6 & 4 & 4 & JG \\
\hline 11 & Aurantii Fructus & 14 & 13 & 12 & ZQ \\
\hline 12 & Linderae Radix & 7 & 6 & 6 & WY \\
\hline 13 & Achyranthis Bidentatae Radix & 13 & 9 & 8 & NX \\
\hline 14 & Cimicifugae Rhizoma & 19 & 14 & 13 & SM \\
\hline 15 & Glycyrrhizae Radix et Rhizoma & 26 & 25 & 25 & GC \\
\hline
\end{tabular}

contribute to its therapeutic effects. In order to obtain the potential active compounds from these herbs of QXH, we applied a method incorporating oral bioavailability (OB), Caco-cell permeability (Caco-2), and drug likeness (DL) evaluation in this work $[20,21]$.

$\mathrm{OB}$ is an important pharmacokinetic parameter which describes the ratio of the amount absorbed into systemic circulation when dosed orally compared to the amount in blood upon intravenous dosing. In the present study, the OB values were evaluated using the robust in silico tool OBioavail1.1 [22]. The compounds with $\mathrm{OB} \geq 30 \%$ were assessed as active compounds for further analysis. Caco- 2 is another vital parameter frequently used as a model for studying the passive diffusion of drugs across the intestinal epithelial barrier. Thus, the in silico Caco-2 permeability model which was constructed by 100 drug molecules with satisfactory statistical results $\left(R^{2}>0.8\right)$ was employed to select compounds that are more likely to possess good permeability [23]. Given those compounds with Caco- 2 value less than -0.4 not permeable, the threshold of Caco-2 permeability is set at -0.4 .

$\mathrm{DL}$ is a qualitative property of chemicals that describes how the pharmacokinetic and pharmaceutical properties of compounds, such as solubility and potency, correspond in the majority of known drugs. In order to identify drug-like compounds, we applied a database-dependent model using the Tanimoto coefficient [24] to calculate the DL (see equation (1)) of each compound in QXH:

$$
T(A, B)=\frac{A \times B}{|A|^{2}+|B|^{2}-A \times B} \text {. }
$$

In equation (1), $A$ represents the molecular descriptors of herbal compounds and $B$ displays the average molecular properties of all compounds in Drugbank. Those compounds with $\mathrm{DL} \geq 0.18$ were preserved. The compounds were adopted as the candidate compounds for further analysis when they met all these three criteria.

Besides, due to the profound pharmacological effects, biological activity, and high contents, those compounds with low OB, Caco-2, or DL values were also selected for further analysis (Table S1). Last but not least, all active compounds were imported into PubChem (https://pubchem.ncbi.nlm. nih.gov) [25] and ChemSpider (http://www.chemspider. com) [26] to standardize their English names.

\subsection{Sample Preparation and UPLC-Q/TOF-MS Verification.}

Reference standards were accurately weighed and directly prepared in methanol as individual stock solutions; subsequently, the stock solutions of twelve standards were continuously diluted with methanol to obtain a suitable concentration for further analysis, respectively. QXH samples were divided into two. $1.0000 \mathrm{~g}$ QXH powder was accurately weighed for each and extracted with methanol $(25 \mathrm{~mL})$ and water $(25 \mathrm{~mL})$ in ultrasonic water bath $(40 \mathrm{kHz}$, $500 \mathrm{~W}$ ) for $30 \mathrm{~min}$ at room temperature, respectively. Subsequently, the QXH methanol and water extracts were centrifuged at $14,000 \mathrm{rpm}$ for $10 \mathrm{~min}$, and the supernatant was stored at $4^{\circ} \mathrm{C}$ until analysis.

The UPLC-Q/TOF-MS system consisted of an Acquity ${ }^{\mathrm{TM}}$ ultra-performance liquid chromatography (UPLC) system (Waters Corporation, Milford, MA, United States) and a Synapt G2 mass spectrometer (MS) (Waters MS-Technologies, Manchester, United Kingdom) equipped with an electrospray ion (ESI) source. The system and data were controlled by MassLynx (V4.1) software. Gradient elution was performed on an ACQUITY UPLC BEH C18 column $(2.1 \mathrm{~mm} \times 100 \mathrm{~mm}, 1.7 \mathrm{~mm}$; Waters $)$ at $35^{\circ} \mathrm{C}$. The optimal mobile phase was composed of $0.1 \%$ formic acid aqueous solution (A) and acetonitrile (B): 0-2 minutes, maintained at $2 \%$ B; $2-4$ minutes, $2-20 \%$ B; $4-5$ minutes, $20-45 \%$ B; $5-8$ minutes, $45-48 \%$; $8-10$ minutes, $48-75 \% \mathrm{~B}$; $10-13$ minutes, 75-90\% B; 13-19 minutes, maintained at 90\% B; 19-22 minutes, 90-95\% B; 22-23 minutes, 95-20\% B; 23-24 minutes, $20-2 \% \mathrm{~B} ; 24-25$ minutes, maintained at $2 \% \mathrm{~B}$. A $2 \mu \mathrm{L}$ aliquot of sample solution was injected for analysis, and the flow rate was $0.4 \mathrm{~mL} / \mathrm{min}$. 
The full-scan LC-MS data were acquired in both positive and negative ion modes from 100 to $1300 \mathrm{Da}$ with a $0.3 \mathrm{~s}$ scan time. The optimal Q/TOF-MS conditions were as follows: capillary voltage $3 \mathrm{kV}$, sampling cone voltage $40.0 \mathrm{~V}$, and extraction cone voltage $4.0 \mathrm{~V}$ for positive ion mode; capillary voltage $2.5 \mathrm{kV}$, sampling cone voltage $40.0 \mathrm{~V}$, and extraction cone voltage $4.0 \mathrm{~V}$ for negative ion mode. The source temperature and desolvation gas temperature were set to 100 and $350^{\circ} \mathrm{C}$, respectively, and the cone gas flow and desolvation gas flow were set to $50 \mathrm{~L} / \mathrm{h}$ and $600 \mathrm{~L} / \mathrm{h}$, respectively. The collision energy was set to $4 \mathrm{eV}$ for positive ion mode and $2 \mathrm{eV}$ for negative ion mode. Sodium formate solution was used to calibrate the mass spectrometer prior to the experiment. Leucine-enkephalin was used as an external reference (Lock-Spray ${ }^{\mathrm{TM}}$ ) to correct the mass during data acquisition via a LockSpray interface, generating reference ions at $m / z 556.2771 \mathrm{Da}\left([M+H]^{+}\right)$in the positive ion mode and $\mathrm{m} / z 554.2615 \mathrm{Da}\left([M-\mathrm{H}]^{-}\right)$in the negative mode.

2.7. Target Prediction. In order to identify the corresponding targets of active compounds in QXH, several methods combined with chemometric strategy, database dependent, information integration, and literature-mining were implemented [27]. Firstly, all active compounds were imported into TCMSP and TCM-MESH again to obtain relevant targets. Subsequently, all active compounds were imported into STITCH (http://stitch.embl.de/) [28], Therapeutic Targets Database (TTD, http://bidd.nus.edu.sg/ group/ttd/) [29], Comparative Toxicogenomics Database (CTD, http://ctdbase.org/) [30], and Google Scholar to obtain $\mathrm{H}-\mathrm{C}-\mathrm{T}$. In view of the preliminary mining results, the active compounds with less targets were introduced into the Similarity Ensemble Approach (SEA, http://sea.bkslab.org/) [31] and Swiss Target Prediction (http://www. swisstargetprediction.ch) [32] to predict their possible targets. Given the prediction results with $\mathrm{Max} \mathrm{TC}<0.57$ and Possibility $<0$ not considered as probably having affinity to the appropriate target, the targets with Max TC $>0.57$ in SEA and Possibility $>0$ in Swiss Target Prediction were kept in the result, respectively. Thereafter, for the purpose of dissecting the mechanisms of the candidate targets in regulating the pathological processes of QS-BSS, the target information was submitted to TTD, CTD, Online Mendelian Inheritance in Man Database (OMIM, http://www.omim. org/) [33], and Uniprot (https://www.uniprot.org) [34] to determine the targets which were related to hemorheological abnormality and coagulopathy, respectively. Finally, all the targets from the previous mining were imported into Uniprot to obtain their corresponding gene symbol, and only the targets of Homo sapiens were kept for further analysis.

2.8. Gene Ontology Enrichment and KEGG Pathway Analysis. The Database for Annotation, Visualization and Integrated Discovery (DAVID, http://david.abcc.ncifcrf.gov) web server was employed to perform gene ontology enrichment and KEGG pathway analysis for the targets related to hemorheological abnormality and coagulopathy, respectively
[35]. Meanwhile, gene ontology enrichment and KEGG pathway analysis were also performed for the overlapped targets involving in the pathological processes of hemorheological abnormality and coagulopathy.

2.9. Network Construction. The herb-active compound-target $\left(\mathrm{H}_{\mathrm{H}^{-}} \mathrm{C}_{\mathrm{H}^{-}}-\mathrm{T}_{\mathrm{H}}\right)$ and herb-active compound-target $\left(\mathrm{H}_{\mathrm{C}^{-}} \mathrm{C}_{\mathrm{C}^{-}} \mathrm{T}_{\mathrm{C}}\right)$ networks of QXH were constructed in the hemorheological abnormality module and coagulopathy module, respectively. All networks were generated and analyzed by Cytoscape 3.6.0 (http://www.cytoscape.org/), an open-source software package project for visualizing, integrating, modeling, and analyzing the interaction networks [36].

2.10. Data Processing and Statistical Analysis. All LC-MS and $\mathrm{MS} / \mathrm{MS}$ data were processed with MassLynx ${ }^{\mathrm{TM}}$ (V4.1, Waters, Manchester, UK) software. Molecular formula speculations of the compounds were determined with Elemental Composition software. Then, the acquired raw data matrices on hemorheology and coagulation function were imported to SIMCA-P (version 14.1, Umetrics, Sweden) for principal component analysis (PCA) and partial least-squares-discriminant analysis (PLS-DA). PCA is an unsupervised multivariate statistical approach used for variable reduction and separation into classes. To maximize class discrimination, the data were further analyzed utilizing PLS-DA $[16,37]$. The parameters of PCA $\left(R^{2} X\right.$ and $\left.Q^{2}\right)$ and PLS-DA $\left(R^{2} X, R^{2} Y\right.$ and $\left.Q^{2}\right)$ were used to evaluate the model, and $Q^{2}$ is an appraisal criterion of the predictive ability of a model. $R^{2} X$ represents the capability wherein the primary components of variables are used to build the model and sample, whereas $R^{2} Y$ represents the conformity level of the sample with the model. Then, the PLS-DA model was validated by 200-repeated permutation tests and CV-ANOVA (analysis of variance testing of cross-validated predictive residuals) tests. A model with the $p$ value (obtained by CV-ANOVA) below 0.5 or with $R^{2}$ and $Q^{2}$ values (obtained by permutation tests) lower than the values of the model itself was considered to be valid. The value of variable importance in the projection (VIP) from the PLS-DA model represents the contribution of variables to the classification of the model which was analyzed. The differential parameters between the control and the model groups were selected by VIP $>1$. All data were expressed as mean \pm standard deviation (SD) and analyzed by one-way ANOVA in SPSS 16.0 (SPSS Inc., Chicago, IL, USA). The criterion for statistical significance was $p<0.05$.

\section{Results}

3.1. Effects of QXH on Hemorheology in Rats with QS-BSS. QS-BSS mainly occurs with increased blood viscosity and poor blood fluidity. WBV and PV are the vital hemorheological parameters and commonly used to evaluate the clinical therapeutic efficacy of drugs. In Table 2, the levels of WBV in low and high shear rates $\left(1 \mathrm{~s}^{-1}, 5 \mathrm{~s}^{-1}, 50 \mathrm{~s}^{-1}, 100 \mathrm{~s}^{-1}\right.$, 
TABLE 2: Effects of QXH on hemorheology in rats with QS-BSS $(\bar{x} \pm s, n=6 \sim 8)$.

\begin{tabular}{|c|c|c|c|c|c|c|c|}
\hline \multirow{2}{*}{ Group } & \multirow{2}{*}{$\begin{array}{c}\text { Doses } \\
\mathrm{g} \times \mathrm{kg}^{-1}\end{array}$} & \multicolumn{5}{|c|}{$\mathrm{WBV}(\mathrm{MPa} \times \mathrm{s})$} & \multirow{2}{*}{ PV } \\
\hline & & $200 \mathrm{~s}^{-1}$ & $100 \mathrm{~s}^{-1}$ & $50 \mathrm{~s}^{-1}$ & $5 s^{-1}$ & $1 \mathrm{~s}^{-1}$ & \\
\hline Control & - & $3.63 \pm 0.10$ & $4.01 \pm 0.15$ & $4.46 \pm 0.20$ & $8.66 \pm 0.50$ & $19.44 \pm 1.39$ & $1.24 \pm 0.07$ \\
\hline Model & - & $4.22 \pm 0.14^{++}$ & $4.70 \pm 0.23^{++}$ & $5.44 \pm 0.29^{++}$ & $14.76 \pm 1.44^{++}$ & $33.61 \pm 3.19^{++}$ & $2.17 \pm 0.43^{++}$ \\
\hline QXH-L & 0.432 & $3.77 \pm 0.17^{* *}$ & $4.07 \pm 0.18^{* *}$ & $4.49 \pm 0.21^{* *}$ & $8.29 \pm 0.48^{* *}$ & $18.07 \pm 1.34^{* *}$ & $1.33 \pm 0.15^{* *}$ \\
\hline QXH-M & 1.296 & $3.93 \pm 0.10^{*}$ & $4.32 \pm 012^{* *}$ & $4.88 \pm 0.17^{* *}$ & $10.11 \pm 0.83^{* *}$ & $24.39 \pm 3.03^{* *}$ & $1.33 \pm 0.15^{* *}$ \\
\hline QXH-H & 2.592 & $3.74 \pm 0.15^{* *}$ & $4.06 \pm 0.18^{* *}$ & $4.54 \pm 0.22^{* *}$ & $8.92 \pm 0.91^{* *}$ & $20.60 \pm 3.24^{* *}$ & $1.42 \pm 0.08^{* *}$ \\
\hline CDDP & 0.1 & $3.84 \pm 0.22^{* *}$ & $4.10 \pm 0.23^{* *}$ & $4.64 \pm 0.38^{* *}$ & $8.39 \pm 0.69^{* *}$ & $17.94 \pm 1.62^{* *}$ & $1.7 \pm 0.28^{* *}$ \\
\hline
\end{tabular}

WBV: whole blood viscosity; PV: plasma viscosity; $200 \mathrm{~s}^{-1}, 100 \mathrm{~s}^{-1}, 50 \mathrm{~s}^{-1}, 5 \mathrm{~s}^{-1}$, and $1 \mathrm{~s}^{-1}$ represent whole blood viscosity in $200 \mathrm{~s}^{-1}, 100 \mathrm{~s}^{-1}, 50 \mathrm{~s}^{-1}, 5 \mathrm{~s}^{-1}$, and $1 \mathrm{~s}^{-1}$ shear rates, respectively. Compared with the control group, ${ }^{+} p<0.05$ and ${ }^{++} p<0.01$; compared with the model group, ${ }^{*} p<0.05$ and ${ }^{* *} p<0.01$.

and $200 \mathrm{~s}^{-1}$ ) and PV were significantly elevated in the model group in comparison with the control group $(p<0.01)$, indicating a successful modeling. Compared with the model group, the WBV at the shear rate of $1 \mathrm{~s}^{-1}, 5 \mathrm{~s}^{-1}, 50 \mathrm{~s}^{-1}, 100 \mathrm{~s}^{-1}$, and $200 \mathrm{~s}^{-1}$ in QXH and CDDP groups significantly decreased near to normality $(p<0.05$ or $p<0.01)$. Meanwhile, QXH and CDDP groups could significantly decrease the rising PV in QS-BSS rats $(p<0.01)$, suggesting that both QXH and CDDP treatment could significantly improve the hemorheological abnormality in rats with QS-BSS, and QXH is no less effective than CDDP in improving hemorheology in QS-BSS rats.

3.2. Effects of QXH on Coagulation Function in Rats with QS$B S S$. APTT, PT, TT, and FIB, as coagulation function test items in clinics, are crucial quantitative indexes of pathological changes in the coagulation system of the body [36]. As is illustrated in Table 3, compared with the control group, TT, PT, and APTT levels were significantly shortened $(p<0.05$ or $p<0.01)$ and FIB content was significantly elevated $(p<0.01)$ in the model group. Compared with the model group, the level of TT, PT, APTT and FIB in QXH groups had different degrees of callback. Specifically, QXH-L treatment could significantly rectify the perturbed TT and PT after QS-BSS modeling $(p<0.05)$; the QXH-M group significantly improved the abnormality of TT and APTT $(p<0.05$ or $p<0.01)$; the QXH-H group showed a significantly effect in improving TT $(p<0.01)$. CDDP significantly brought the disrupted TT, APTT, and FIB after QS-BSS modeling to near normality $(p<0.05$ or $p<0.01)$. All above results fully prove that $\mathrm{QXH}$ is comparable with CDDP on improving coagulation function in QS-BSS rats.

\subsection{Evaluation of the Effects of QXH on Improving Hemor-} heological Abnormality and Coagulopathy Based on Multivariate Statistical Analysis. In this study, PCA was used firstly to determine the general interrelation between control, model, QXH-L, QXH-M, QXH-H, and CDDP groups. As is shown in Figure S1, the model group and other groups were separated clearly, indicating that the rats of the model group have significantly altered on hemorheology and coagulation function. The parameters of the PCA model: $R^{2} X=0.982 ; Q^{2}=0.582$ (Figure $S 1$ ) suggesting good ability of prediction and reliability of the model, and the variances of Comp [1] and Comp [2] account for 0.61 and 0.125, respectively. To provide better visualization for discriminating groups of samples from PCA and for class-separating information of the variables, a supervised approach (PLS-DA) was performed. Obviously, in a PLS-DA score plot (Figure 2), the center of the control group is about $(1.69,-0.4)$, the center of the model group is about $(-4.36,-0.61)$, the center of the QXH-L group is about $(0.67,-0.46)$, the center of the QXH-M group is about $(-0.65,1.07)$, the center of the QXH-H group is about $(0.61,0.08)$, and the center of the CDDP group is about $(1.03,0.38)$. By the analysis of central distance among groups, it was suggested that the QXH-L and QXH-H groups have a better pharmacodynamic effect on QS-BSS. Meanwhile, the CDDP group also shows powerful regulation on rats with QS-BSS to the normal level. The parameters of the PLS-DA model: $R^{2} X=0.72$, $R^{2} Y=0.245$, and $Q^{2}=0.164$ (Figure 2(a)), suggesting the goodness of fit to data. And the variances of Comp [1] and Comp [2] in Figure 2 account for 0.609 and 0.11, respectively. Permutation tests $(n=200)$ and CV-ANOVA tests were used to validate the PLS-DA model among all groups (Figure S2) and the PLS-DA model with $R^{2}=(0.0,-0.0123)$, $Q^{2}=(0.0,-0.191)$, and $p=0.000490249$, indicating that the PLS-DA model is valid.

Subsequently, the second round of the PLS-DA method was used to analyze the VIP value of each index between control and model groups to obtain the most contribution indexes to the two groups' differences. The parameters of PLS-DA between control and model groups: $R^{2} X=0.884$, $R^{2} Y=0.918$, and $Q^{2}=0.87$. These results indicated the excellent reliability and predictive capability of the model (Figure S3). The results of 200 permutations tests and CVANOVA tests on the PLS-DA model with $R^{2}=(0.0,0.27)$, $Q^{2}=(0.0,-0.196)$, and $p=0.000174217$ (Figures S4) indicate the PLS-DA model is valid. As is shown in Figure 2(b), the value of VIP from the PLS-DA model represents the contribution of variables of the indexes to the classification between control and model groups. The larger the VIP value, the greater the contribution of those indexes to the difference between control and model groups. The VIP value of WBV5 $\mathrm{s}^{-1}, \mathrm{WBV} 1 \mathrm{~s}^{-1}$, WBV200 s $\mathrm{s}^{-1}, \mathrm{FIB}$, and WBV50 s ${ }^{-1}$ are all $>1$, indicating WBV and FIB are vital to distinguish the difference between normal and QS-BSS levels and can be used as the essential indexes to evaluate the pharmacodynamic effect of QXH in the treatment of QS-BSS. The VIP value of TT and APTT are about 0.9 , suggesting the poor contribution to the difference between control and model groups. 
TABLE 3: Effects of QXH on coagulation function in rats with QS-BSS $(\bar{x} \pm s, n=6 \sim 8)$.

\begin{tabular}{|c|c|c|c|c|c|}
\hline \multirow{2}{*}{ Group } & \multirow{2}{*}{$\begin{array}{c}\text { Doses } \\
\mathrm{g} \times \mathrm{kg}^{-1}\end{array}$} & \multicolumn{4}{|c|}{ Coagulation function } \\
\hline & & TT (s) & $\mathrm{PT}(\mathrm{s})$ & APTT (s) & FIB $(\mathrm{g} / \mathrm{L})$ \\
\hline Control & - & $25.34 \pm 1.14$ & $12.21 \pm 1.17$ & $21.45 \pm 2.10$ & $2.94 \pm 0.40$ \\
\hline Model & - & $21.70 \pm 1.51^{+}$ & $9.25 \pm 0.38^{++}$ & $16.64 \pm 0.96^{++}$ & $4.81 \pm 0.47^{++}$ \\
\hline QXH-L & 0.432 & $25.21 \pm 1.69^{*}$ & $12.03 \pm 2.28^{*}$ & $18.24 \pm 2.66$ & $3.93 \pm 0.81$ \\
\hline QXH-M & 1.296 & $27.98 \pm 0.56^{* *}$ & $9.96 \pm 0.847$ & $21.78 \pm 3.10^{*}$ & $4.12 \pm 0.64$ \\
\hline QXH-H & 2.592 & $28.20 \pm 1.29^{* *}$ & $11.22 \pm 1.25$ & $19.05 \pm 1.90$ & $4.30 \pm 0.73$ \\
\hline CDDP & 0.1 & $24.36 \pm 3.74^{* *}$ & $11.04 \pm 1.35$ & $25.5 \pm 1.33^{* *}$ & $3.64 \pm 0.50^{*}$ \\
\hline
\end{tabular}

TT: thrombin time; PT: prothrombin time; APTT: activated partial thromboplastin; FIB: fibrinogen content. Compared with the control group, ${ }^{+} p<0.05$ and ${ }^{++} p<0.01$; compared with the model group ${ }^{*} p<0.05$ and ${ }^{* *} p<0.01$.

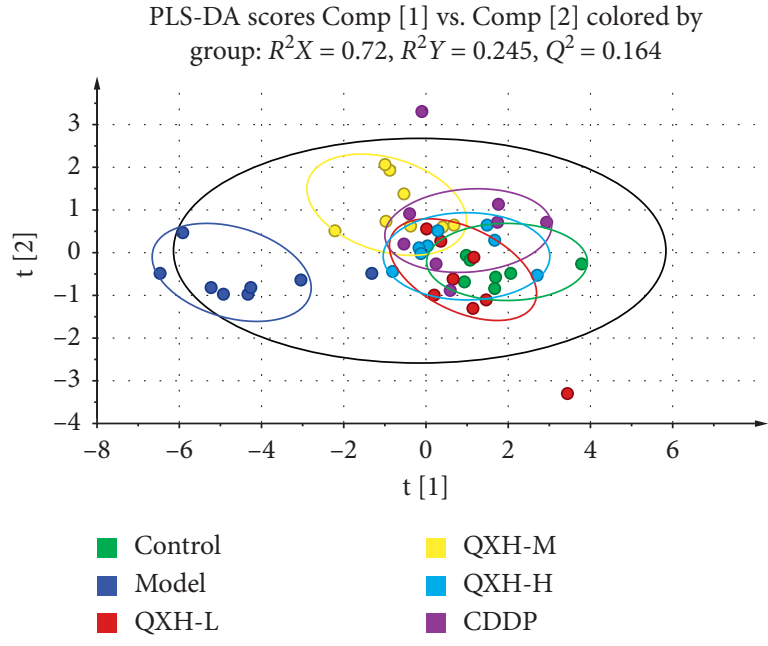

(a)

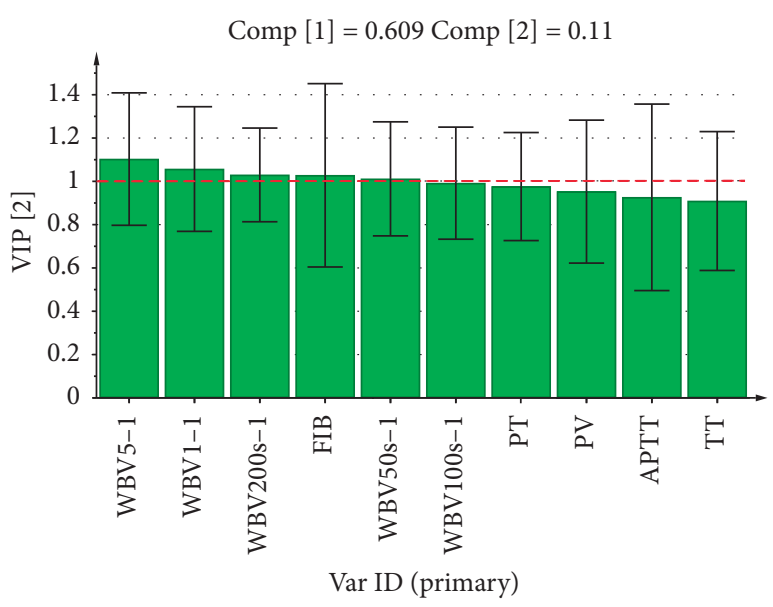

(b)

FIgURE 2: (a) PLS-DA score plot of hemorheology and coagulation function indexes among all groups. (b) VIP score plot of hemorheology and coagulation function indexes between control and model groups. WBV200 s ${ }^{-1}$ : whole blood viscosity in $200 \mathrm{~s}$ shear rate; WBV100 s whole blood viscosity in $100 \mathrm{~s}$ shear rate; WBV50 $\mathrm{s}^{-1}$ : whole blood viscosity in $50 \mathrm{~s}$ shear rate; WBV $5 \mathrm{~s}^{-1}$ : whole blood viscosity in $5 \mathrm{~s}$ shear rate; WBV $1 \mathrm{~s}^{-1}$ : whole blood viscosity in $1 \mathrm{~s}$ shear rate; PV: plasma viscosity; APTT: activated partial thromboplastin time; PT: prothrombin time; TT: thrombin time; FIB: fibrinogen content.

3.4. Active Compounds of QXH. To better understand the molecular mechanisms behind the therapeutic effects of QXH, a total of 195 active compounds were selected for further analysis, 25 of which were verified in QXH by UPLC-Q/TOF-MS, such as tetrahydropalmatine (S1), berberine (S2), butylated hydroxytoluene (S3), tangeretin (S4), $\beta$-sitosterol (S5), Z-ligustilide (S6), cryptotanshinone (S7), tanshinone I (S8), levistilide A (S9), tanshinone IIA (S10), stigmasterol (S11), oxypaeoniflorin (S12), amygdalin (13), caffeic acid (S14), albiflorin (S15), paeoniflorin (S16), liquiritin (S17), ferulic acid (S18), isoferulic acid (S19), rosmarinic acid (S20), liquiritigenin (S21), benzoylpaeoniflorin (S22), glycyrrhizic acid (S23), isoliquiritigenin (S24), and glycyrrhetic acid (S25) (Table S1, Figures S5 and S6). Especially, butylated hydroxytoluene, berberine, tangeretin, quercetin, luteolin, columbamine, ellagic acid, and tanshinone IIB are the major agents of QXH, which play anti-inflammation, antiatherosclerotic, antiplatelet aggregation, and antithrombotic effects, respectively $[38,39]$. It was worth noting that although several compounds have relatively low pharmacokinetic values, they were either the richest compounds of the herbs or biologically active, like paeonolide, danshensu, and rosmarinic acid; thus, those compounds were also considered as candidate compounds for further analysis. In other words, there were 19 compounds in Angelicae Sinensis Radix (DG), 17 compounds in Paeoniae Radix Rubra (CS), 15 compounds in Chuanxiong Rhizoma (CX), 12 compounds in Persicae Semen (TR), 15 compounds in Carthami Flos (HH), 11 compounds in Bupleuri Radix (CH), 11 compounds in Cyperi Rhizoma (XF), 29 compounds in Salviae Miltiorrhizae Radix et Rhizoma (DS), 33 compounds in Corydalis Rhizoma (YHS), 6 compounds in Platycodonis Radix (JG), 14 compounds in Aurantii Fructus (ZQ), 7 compounds in Linderae Radix (WY), 13 compounds in Achyranthis Bidentatae Radix (NX), f 19 compounds in Cimicifugae Rhizoma (SM), and 26 compounds in Glycyrrhizae Radix et Rhizoma (GC). Zligustilide, senkyunolide A, senkyunolide I, 3-butylidenephthalide, coniferyl ferulate, vanillin, erulic acid, and sedanolide were shared in DG and CX. Quercetin was shared in $\mathrm{HH}, \mathrm{NX}, \mathrm{WY}, \mathrm{YHS}, \mathrm{CH}, \mathrm{XF}$, and ZQ. Rosmarinic acid was shared in $\mathrm{HH}$ and DS. Kaempferol was shared in $\mathrm{HH}, \mathrm{NX}$, $\mathrm{CH}, \mathrm{XF}$, and $\mathrm{ZQ}$. 
3.5. Target Identification. Presently, for QXH, altogether 102 proteins were identified as its targets, and 51 of 102 targets were involved in hemorheological abnormality module, 68 of 102 targets were involved in coagulopathy module, and 17 targets were shared in improving hemorheological abnormality and coagulopathy. In the hemorheological abnormality module, PTGS1, PTGS2, AR, ESR1, ESR2, CHRM5, PIK3CG, MAPK14, CYP3A4, and HMGCR were included. PTGS1 and PTGS2, two isoforms of cyclooxygenase, are the anti-inflammation targets of most NSAIDs [40], PTGS1 is constitutively expressed in various cells and tissues, fulfilling housekeeping functions, such as vascular homeostasis and platelet aggregation [41]. PTGS2 is an immediate-early response gene induced in response to mitogens, tumor promoters, cytokines, growth factors, and inflammatory stimuli [42]. ESR1 is mainly expressed in macrophages, endothelial cells, and vascular smooth muscle cells, and it plays an important role in vascular wall physiology and function. Single-nucleotide polymorphisms in the ESR1 gene have been associated with vascular diseases and conditions, such as arterial hypertension, CVD, cerebrovascular disease, and serum lipid level alterations [43]. In the coagulopathy module, besides PTGS1, PTGS2, and PIK3CG, F2, F10, PRKACA, NOS3, PTPN2, NQO1, and HMOX1 were also included. F2 and F10 cleave bonds after Arg and Lys, convert fibrinogen to fibrin and activate factors V, VII, VIII, and XIII, in combination with thrombomodulin and protein $\mathrm{C}$, and has function in blood homeostasis, inflammation, and wound healing [44]. NOS3 promotes the production of NO; NO can mediate vascular endothelial growth factor- (VEGF) induced angiogenesis in coronary vessels and promote blood clotting through the activation of platelets [45].

3.6. $H_{H^{-}} C_{H^{-}} T_{H}$ Network of QXH (Hemorheological Abnormality Module). Hemorheological abnormality is an important part of the formation of QS-BSS, which is characterized by changes in blood fluidity, viscosity, deformability, and coagulability. Thus, we constructed a $\mathrm{H}_{\mathrm{H}^{-}}$ $\mathrm{C}_{\mathrm{H}}-\mathrm{T}_{\mathrm{H}}$ network through network analysis to illuminate the relationship among herbs, active compounds, and candidate targets (Figure 3 (for original picture, see Figure S7)). This network consists of 251 nodes (15 herbs, 185 active compounds, and 51 candidate targets; Tables S2 and S3). As is depicted in the $\mathrm{H}_{\mathrm{H}}-\mathrm{C}_{\mathrm{H}}-\mathrm{T}_{\mathrm{H}}$ network, red, green, and blue nodes represent herbs, active compounds, and candidate targets, respectively. The size of the nodes is positively related to the value of degree. Degree, which is the number of edges connected to the node, and the betweenness, another centrality index defined by the number of times a node acts a bridge along the shortest path between two other nodes, was calculated. Actually, betweenness reflects the fraction of the shortest paths in the network that pass through any particular node and a measure of the importance of a node as a hub in a network [46]. To gain insights into the pharmacological mechanisms of QXH on hemorheological abnormality, we performed gene ontology enrichment and KEGG pathway analysis on the 51 relevant targets, and the top 10 of biological process, molecular function, and cellular component and 28 KEGG pathways were predicted (Figure 4). The results of biological process were mainly enriched in regulation of cholesterol storage, lipoprotein metabolic process, blood circulation, and regulation of lipid storage, which were consistent with the pathologic mechanisms of hemorheological abnormality.

The degree and betweenness of the top 10 candidate compounds and targets involvied in hemorheological abnormality module are shown in Tables 4 and 5. Among the active compounds, quercetin, luteolin, isorhamnetin, butylated hydroxytoluene, and isoliquiritigenin were the top ones, interacting with $30,13,13,12$, and 12 targets, respectively. Quercetin acted on PTGS1, PTGS2, ALOX15, and PPARA and participated in the PPAR signaling pathway, prolactin signaling pathway, and VEGF signaling pathway. Numerous studies have shown that quercetin plays an important role in anti-inflammatory, antiproliferative, and antiatherosclerotic, especially in reducing blood fat and increasing coronary blood flow, which was contained by $\mathrm{HH}, \mathrm{TR}, \mathrm{JG}, \mathrm{WY}, \mathrm{CH}$, and ZQ $[40,47]$. Luteolin acting on PTGS1, PTGS2, AR, and APP could depress hypertensive aorta remodeling in spontaneous hypertensive rats, relax porcine coronary and aortic arteries, and improve cardiac function after myocardium ischemia/reperfusion injury $[41,42]$, which is vital to the treatment of cardiovascular diseases contained by $\mathrm{HH}, \mathrm{JG}$, $\mathrm{XF}$, and ZQ. Butylated hydroxytoluene acting on PTGS1, PTGS2, AR, ESR1, and CYP3A4 is an antioxidant that has been shown to have an antiatherosclerotic effect in normal cholesterol-fed rabbits, which was contained by CS [48]. Kaempferol acting on AR, PIK3CG, ESR1, ESR2, CYP3A4, and APOE was involved in regulation of lipolysis in adipocytes, prolactin signaling pathway, estrogen signaling pathway, arachidonic acid metabolism, and VEGF signaling pathway, which is an important active member of dietary flavonoids discovered in many kinds of fruits and vegetables. Che et al. [49] demonstrated that kaempferol could effectively protect human umbilical vein endothelial cells against oxidative stress induced by ox-LDL, which indicated that kaempferol is capable of preventing atherosclerotic vascular disease. Rosmarinic acid acting on PTGS2, AR, ESR1, IL4, and NR1H4 was the active compound in DS and $\mathrm{HH}$, which could inhibit the formation of malondialdehyde in human platelets in vitro, and its IC50 value is $3.37 \mathrm{nmol} / \mathrm{L}$, indicating that rosmarinic acid has antiplatelet aggregation activity [50]. Berberine is a new cholesterol-lowering drug, and studies have shown that oral administration of berberine in 32 hypercholesterolemia patients for 3 months reduces serum cholesterol and triglycerides by $29 \%$ and $35 \%$, respectively [51, 52].

Obviously, though the analysis of hemorheological abnormality module revealed that quercetin, luteolin, isorhamnetin, butylated hydroxytoluene, isoliquiritigenin, nicotinic acid, kaempferol, rosmarinic acid, campesterol, and berberine may play an important role in improving hemorheological abnormality, the hub targets of QXH in the treatment of QS-BSS were PTGS2, PTGS1, AR, ESR1, CHRM5, ESR2, MMP2, PIK3CG, CYP3A4, and HMGCR. 


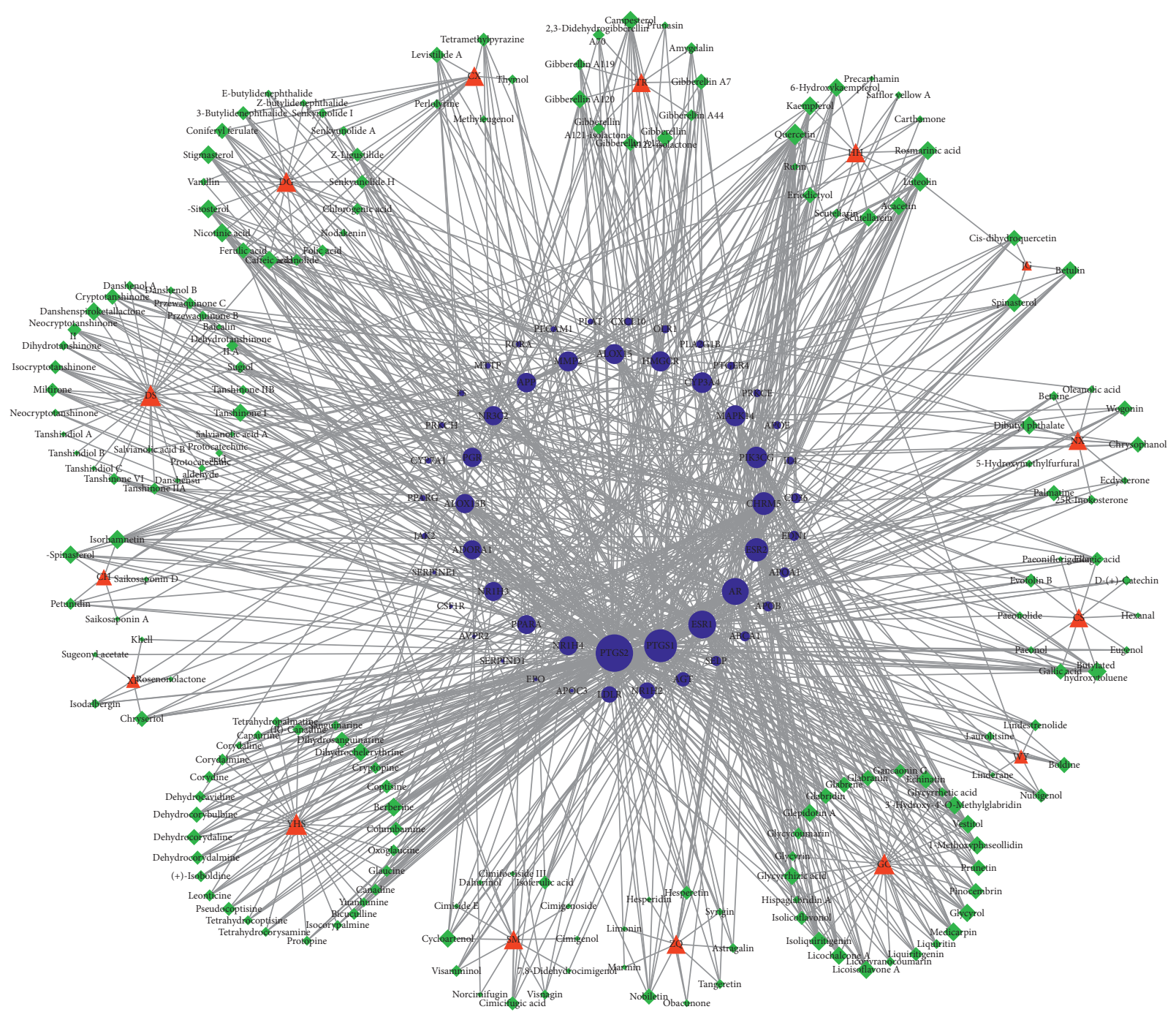

Figure 3: The $\mathrm{H}_{\mathrm{H}}-\mathrm{C}_{\mathrm{H}^{-}}-\mathrm{T}_{\mathrm{H}}$ network of QXH (hemorheological abnormality module; red node: herbs, green node: compounds, and blue node: targets).

Quercetin was the active compound of $\mathrm{HH}, \mathrm{WY}, \mathrm{CH}$, and ZQ; luteolin was the active compound of $\mathrm{HH}$, JG, XF, and ZQ; isorhamnetin was only contained by $\mathrm{CH}$; butylated hydroxytoluene was contained by CS; nicotinic acid was the active compound in DG; rosmarinic acid was contained by DS and $\mathrm{HH}$; and campesterol and berberine were contained by TR and YHS, respectively. Kaempferol was the active compound of $\mathrm{HH}, \mathrm{CH}$, and $\mathrm{ZQ}$. Based on this, we can infer that $\mathrm{HH}, \mathrm{CS}, \mathrm{DG}, \mathrm{TR}, \mathrm{YHS}, \mathrm{CH}$, and ZQ were exerting effects for improving hemorheological abnormality.

KEGG analysis showed that the targets (related to hemorheological abnormality) mainly involved in PPAR signaling pathway, fat digestion and absorption, arachidonic acid metabolism, prolactin signaling pathway, inflammatory mediator regulation of TRP channels, regulation of lipolysis in adipocytes, and TNF signaling pathway.

3.7. $H_{C^{-}} C_{C}-T_{C}$ Network of $\mathrm{QXH}$ (Coagulopathy Module). Interestingly, quercetin, isorhamnetin, luteolin, kaempferol, berberine, wogonin, and isoliquiritigenin were not only playing a significant role in regulating hemorheological abnormality but also exerting effects for improving coagulopathy (Table 4). In Figure 5 (original picture see Figure S8), this network consisted of 267 nodes (15 herbs, 184 candidate compounds, and 68 candidate targets, Tables S2 and S3). The degree and betweenness of the top 10 candidate compounds and targets involved in improving coagulopathy are shown in Tables 4 and 5. Tangeretin was one of the active compounds of ZQ, which interacted with the hub targets (such as PTGS1, PTGS2, IL6, F2, F10, NQO1, and HMOX1) in coagulation function module. Columbamine can significantly reduce the levels of TC, TG, and LDL-c in the serum of golden hamsters (HFHC) induced by high fat and high cholesterol, while increasing the level of HDL-c [53]. Ellagic acid was one of the main active compounds of CS, which acted on HMOX1, NQO1, F12, SYK, and NOS3, and was involved in PI3K-Akt signaling pathway and platelet activation. Tanshinone IIB acted on F2, PTGS2, MPI , and other targets with antiplatelet aggregation activity [54]. Betaine was proved to protect against coagulation events in vivo and vitro, decreasing lipid peroxidation in plasma [55]. 


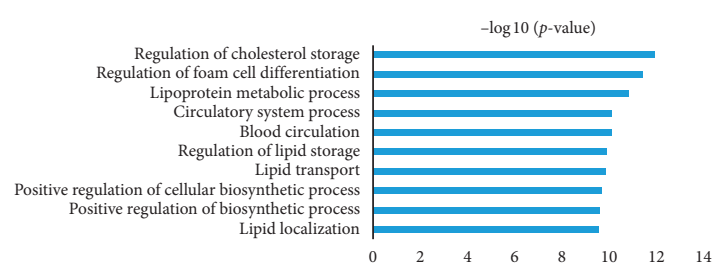

(a)

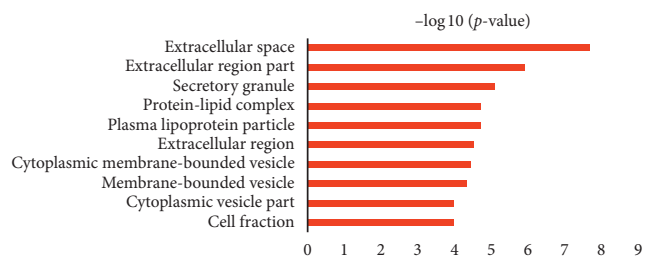

(c)

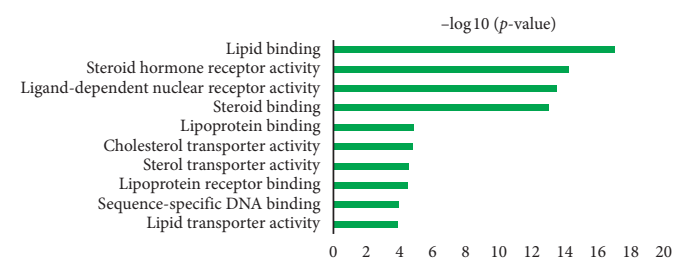

(b)

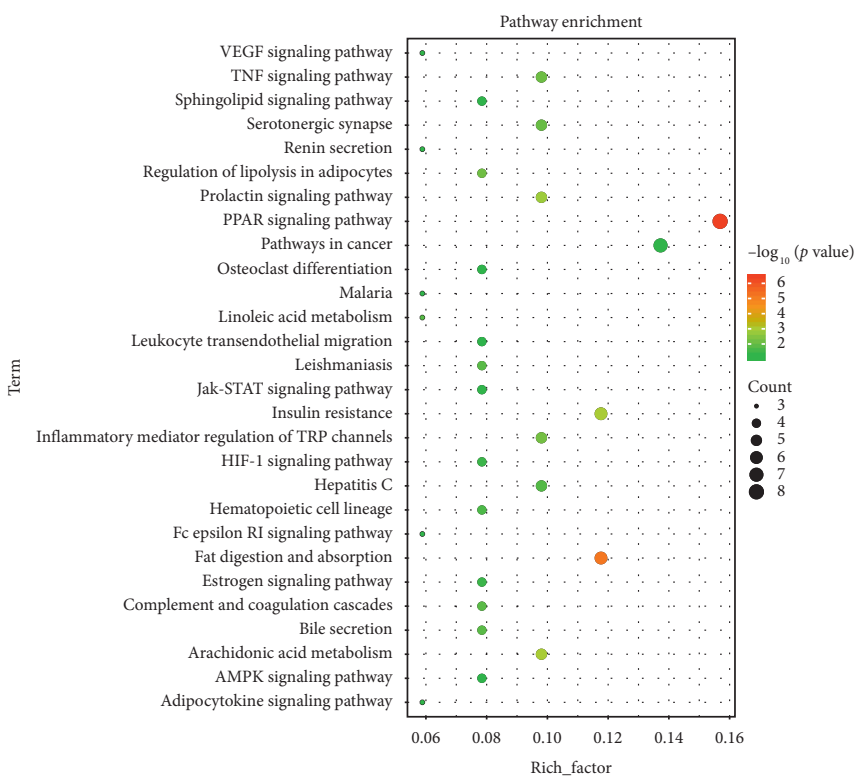

(d)

FIGURE 4: Gene ontology enrichment and KEGG pathway analysis of the targets of QXH related to hemorheological abnormality module. (a) Biological process. (b) Molecular function. (c) Cellular component. (d) KEGG pathway.

TABLE 4: Top 10 candidate compounds (hemorheological abnormality module and coagulopathy module) according to 2 centrality indexes.

\begin{tabular}{lccc}
\hline Compounds & Degree & Compounds & Betweenness \\
\hline Hemorheological abnormality & & & \\
Quercetin & 32 & Quercetin & 0.0857877 \\
Luteolin & 16 & Luteolin & 0.02594642 \\
Isorhamnetin & 13 & Butylated hydroxytoluene* & 0.01562293 \\
Butylated hydroxytoluene* & 12 & Isorhamnetin & 0.01488112 \\
Isoliquiritigenin & 12 & Isoliquiritigenin* & 0.01377775 \\
Nicotinic acid & 12 & Gallic acid & 0.01187844 \\
Kaempferol & 12 & Berberine* & 0.01178984 \\
Rosmarinic acid* & 11 & Nicotinic acid & 0.01170317 \\
Campesterol & 11 & Glycyrrhizic acid* & 0.01089196 \\
Berberine* & 10 & Rosmarinic acid* & 0.01077513 \\
Coagulopathy & & & \\
Quercetin & 34 & Quercetin & 0.0827979 \\
Isorhamnetin & 16 & Luteolin & 0.0211428 \\
Luteolin & 14 & Wogonin & 0.0211385 \\
Kaempferol & 14 & Isorhamnetin & 0.020552 \\
Tangeretin* & 13 & Ellagic acid & 0.0161838 \\
Berberine* & 13 & Tanshinone IIB & 0.0145945 \\
Columbamine & 11 & Betaine & 0.0142141 \\
Wogonin & 11 & Berberine* & 0.0130752 \\
Z-ligustilide* & 10 & Ferulic acid* & 0.0130271 \\
Ferulic acid* & 10 & Tangeretin* & 0.0124719 \\
\hline
\end{tabular}

The active compounds which had been verified by UPLC-Q/TOF-MS in QXH were labeled with *. 
TABle 5: Top 10 candidate targets (hemorheological abnormality module and coagulopathy module) according to 2 centrality indexes.

\begin{tabular}{|c|c|c|c|}
\hline Targets & Degree & Targets & Betweenness \\
\hline \multicolumn{4}{|c|}{ Hemorheological abnormality } \\
\hline PTGS2 & 149 & PTGS2 & 0.39630531 \\
\hline PTGS1 & 115 & PTGS1 & 0.1906698 \\
\hline ESR1 & 77 & $\mathrm{AR}$ & 0.09648379 \\
\hline $\mathrm{AR}$ & 70 & ESR1 & 0.09300286 \\
\hline ESR2 & 43 & CHRM5 & 0.03405704 \\
\hline CHRM5 & 39 & ESR2 & 0.02949908 \\
\hline PIK3CG & 29 & MMP2 & 0.02707469 \\
\hline MAPK14 & 25 & PIK3CG & 0.01870076 \\
\hline CYP3A4 & 23 & CYP3A4 & 0.0143101 \\
\hline HMGCR & 19 & HMGCR & 0.01310125 \\
\hline \multicolumn{4}{|c|}{ Coagulopathy } \\
\hline PTGS2 & 149 & PTGS2 & 0.4147934 \\
\hline PTGS1 & 115 & PTGS1 & 0.1977836 \\
\hline F10 & 45 & IL6 & 0.0557075 \\
\hline PRKACA & 45 & F2 & 0.0542773 \\
\hline $\mathrm{F} 2$ & 40 & F10 & 0.0379244 \\
\hline NOS3 & 34 & NOS3 & 0.0275241 \\
\hline IL6 & 33 & PRKACA & 0.0275035 \\
\hline PIK3CG & 29 & PTPN2 & 0.0243642 \\
\hline PTPN2 & 28 & NQO1 & 0.0201226 \\
\hline ADRA2C & 28 & HMOX1 & 0.0175608 \\
\hline
\end{tabular}

Remarkably, multiple molecular targets interacted with the bioactive compounds (Tables 4, and 5 and Figure 5), such as PTGS1, PTGS2, F10, PRKACA, F2, NOS3, IL6, PIK3CG, PTPN2, and ADRA2C. And 68 targets with relevant biological process demonstrated a closed relation to coagulation function. In platelets, PTGS1 and PTGS2 are involved in the generation of thromboxane A2, which promote platelet activation and aggregation, vasoconstriction, and proliferation of vascular smooth muscle cells [56].

Tangeretin was the active compound in ZQ. Ferulic acid was shared in DG, CX, and SM. Ellagic acid was only contained by CS. Columbamine was the active compound in YHS, and wogonin was contained by NX. Hence, we can infer that ZQ, CX, and SM also contributed to improving coagulopathy in addition to HH, DG, CS, TR, and YHS.

KEGG analysis (Figure 6) showed that the targets (related to coagulopathy) were mainly involved in complement and coagulation cascades, VEGF signaling pathway, vascular smooth muscle contraction, calcium signaling pathway, arachidonic acid metabolism, and leukocyte transendothelial migration. Over recent years, several studies have linked complement and thrombosis to the complement and coagulation cascades [57]. The thrombosis consists of insoluble fibrin, deposited platelets, accumulated white blood cells, and trapped red blood cells, which lead to the formation of blood stasis syndrome. VEGF signaling pathway leads to endothelial cell apoptosis, and a study has shown that the genetic repression of mouse VEGF expression regulates coagulation cascade [58]. Moreover, calcium signaling pathway plays a vital role in the development of platelet activation [59].
3.8. Analysis of the Overlapped Compounds, Targets, and Pathways in the Pathological Processes of Hemorheological Abnormality and Coagulopathy. By analysis of the overlapped compounds, targets, and pathways involved in hemorheological abnormality and coagulopathy, 174 of 195 active compounds, 17 of 102 targets (Table S4), and 6 of 50 pathways associated with QXH were shared in the improvement of hemorheological abnormality and coagulopathy, indicating QXH in treating QS-BSS by the synergy of multiple compounds, targets, and pathways (Figure 7). Then, the biological process of overlapped targets was analyzed, which indicated that the pathological processes of hemorheological abnormality and coagulopathy were involved in inflammatory response, response to oxidative stress, cyclooxygenase pathway, angiogenesis, immune system process, regulation of blood pressure, and response to hypoxia of total 6 biological processes. Hence, in addition to hemorheological abnormality and coagulopathy, the participation of inflammation and immunity in the formation of QS-BSS is also worthy of our attention. Complement and coagulation cascades, arachidonic acid metabolism, VEGF signaling pathway, leukocyte transendothelial migration, Fc epsilon RI signaling pathway, and pathways in cancer might be the hub pathways of QXH in treating QS-BSS, which were shared in improving hemorheological abnormality and coagulopathy.

\section{Discussion}

TCM is a precious heritage which has been practiced for thousands of years in China and surrounding countries. Nowadays, herbal medicine has been widely used for complicated disease treatment and is gradually becoming acceptable as an alternative medicine worldwide [60]. TCM network pharmacology was a new research strategy for translating TCM from an experience-based medicine to an evidence-based medicine system, which predicted the target profiles and pharmacological actions of herbal compounds and revealed drug-gene-disease comodule associations to interpret the combinatorial rules and network regulation effects of herbal formula [61]. Therefore, we herein utilized a combination method that included pharmacodynamics evaluation and network pharmacology to elucidate the pharmacodynamic effect, substances, and potential mechanisms of QXH through improving hemorheology and coagulation function in treating QS-BSS.

Hemorheology and coagulation function provide an important approach for studies on the mechanisms of QSBSS and the therapeutic effects of Chinese herbal compounds that promote blood circulation and remove blood stasis [16]. In our work, we established the QS-BSS model in rats to evaluate the pharmacodynamic effect of QXH in improving hemorheological abnormality and coagulopathy. CDDP, one Chinese patent drug, was used as the positive control drug in this study. Preclinical and clinical studies have suggested that CDDP can expand blood vessel, promote blood circulation, relieve blood stasis, improve microcirculation, and improve hemorheological property [62]. The results of pharmacodynamic evaluation showed that 


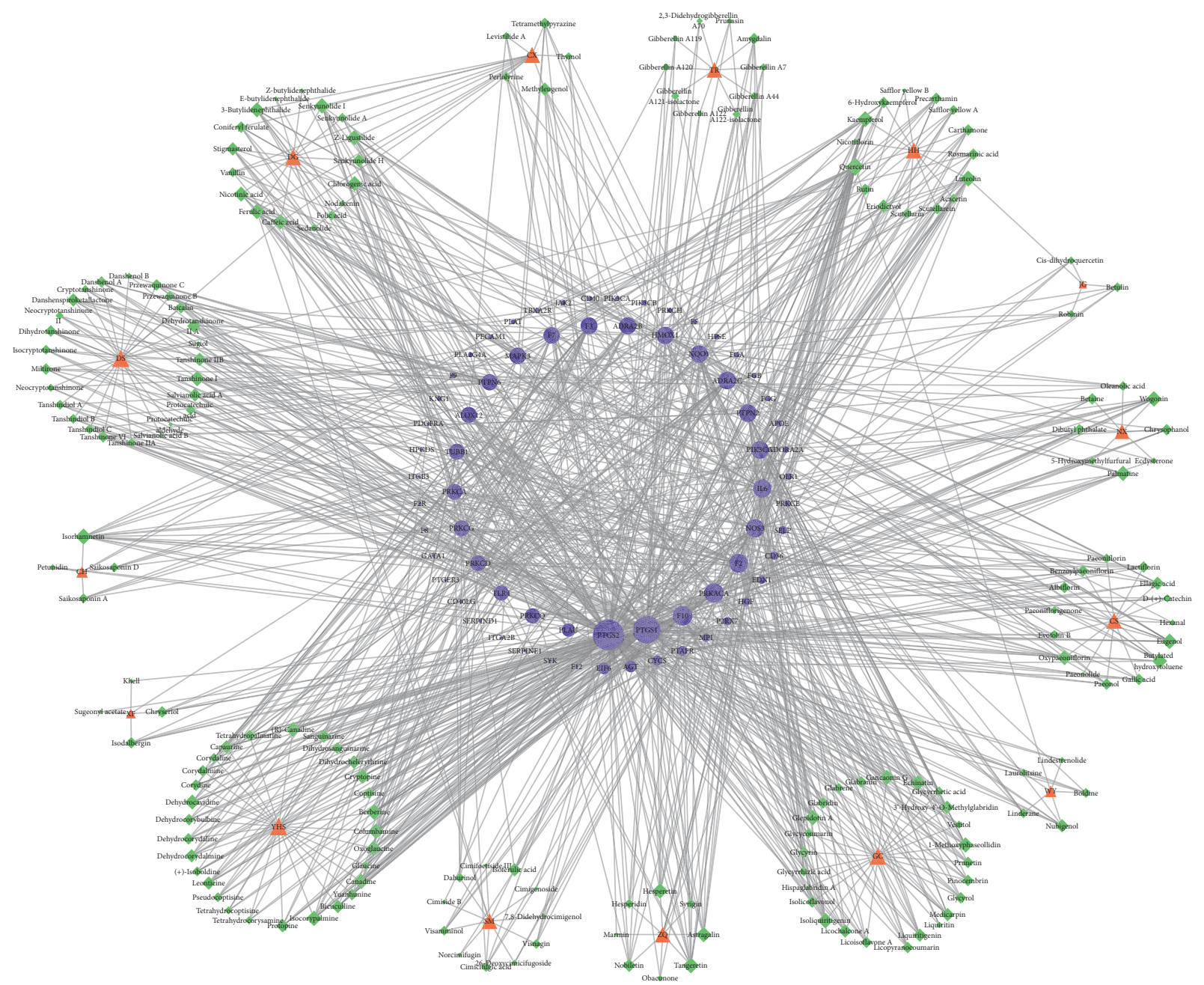

Figure 5: The $\mathrm{H}_{\mathrm{C}}-\mathrm{C}_{\mathrm{C}}-\mathrm{T}_{\mathrm{C}}$ network of QXH (coagulopathy module, red node: herbs, green node: compounds, and blue node: targets).

QXH and CDDP groups significantly regulated the hemorheological abnormality and coagulopathy in rats with QS-BSS. Especially, QXH-L and QXH-H groups could significantly improve the abnormal increase of WBV and PV in rats with QS-BSS and had different degrees of callback on APTT, TT, PT, and FIB, which are comparable with CDDP in improving hemorheology and coagulation function. In terms of PCA and PLS-DA, after the preadministration of QXH, the same parameters in the QS-BSS model were close to those in the control and CDDP groups, also indicating that QXH has significant pharmacodynamic effect on QS-BSS. All of the above results were consistent with those of previous studies [63-65]. Hence, QXH, through promoting blood circulation and removing blood stasis in treating QS-BSS, gives us a glance at TCM compound formula in the treatment of the disease with a variety of pathogenesis by the synergy of various herbal medicines.

Thereby, we performed a network pharmacology approach which contains $\mathrm{OB}, \mathrm{Caco}-2$, and DL screening, active compound verification, target prediction, and network analysis to dissect the active compounds and possible mechanisms of QXH on regulating hemorheological abnormality and coagulopathy. The main results are as follows:
(1) 195 active compounds in 15 herbs and 102 targets were identified as candidate targets related to QS-BSS. These active compounds provided clues to our study of the substance on the basis of the pharmacodynamic effect of QXH. (2) Meanwhile, 25 active compounds were verified by UPLC-Q/TOF-MS on QXH methanol and water extracts, which are corresponding to the results of network pharmacology. (3) Through the network analysis of QXH on the two vital pathological processes of QS-BSS, the mechanism of QXH in the treatment of QS-BSS is gradually clear.

In the hemorheological abnormality network module, 51 targets participated in signaling pathways such as PPAR signaling pathway, prolactin signaling pathway, fat digestion and absorption, estrogen signaling pathway, VEGF signaling pathway, and leukocyte transendothelial migration. A recent study on antihyperlipidemic activity of quercetin (included in $\mathrm{HH}, \mathrm{NX}, \mathrm{WY}, \mathrm{YHS}, \mathrm{CH}, \mathrm{XF}$, and ZQ) revealed that it may be involved in regulating the PPAR signaling pathway [66], which is comparable to our findings. The PPAR signal pathway is concerned with the formation of QS-BSS. PPAR $\gamma$ is highly expressed in macrophage-derived foam cells of both early and advanced atherosclerotic lesions [67]. PPAR $\gamma$ ligands may attenuate inflammation and hence, 


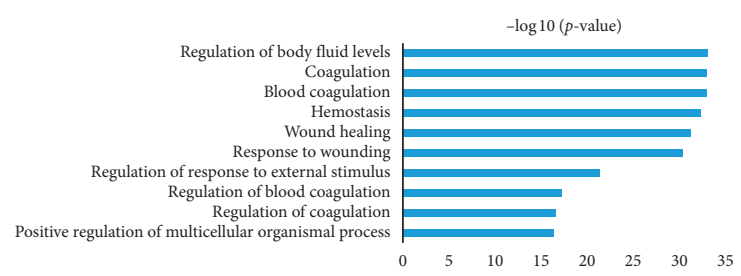

(a)

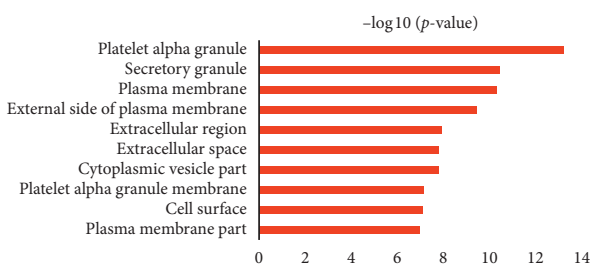

(c)

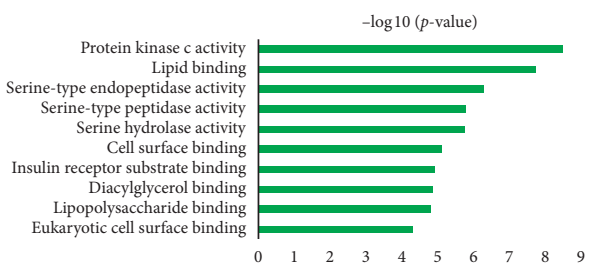

(b)

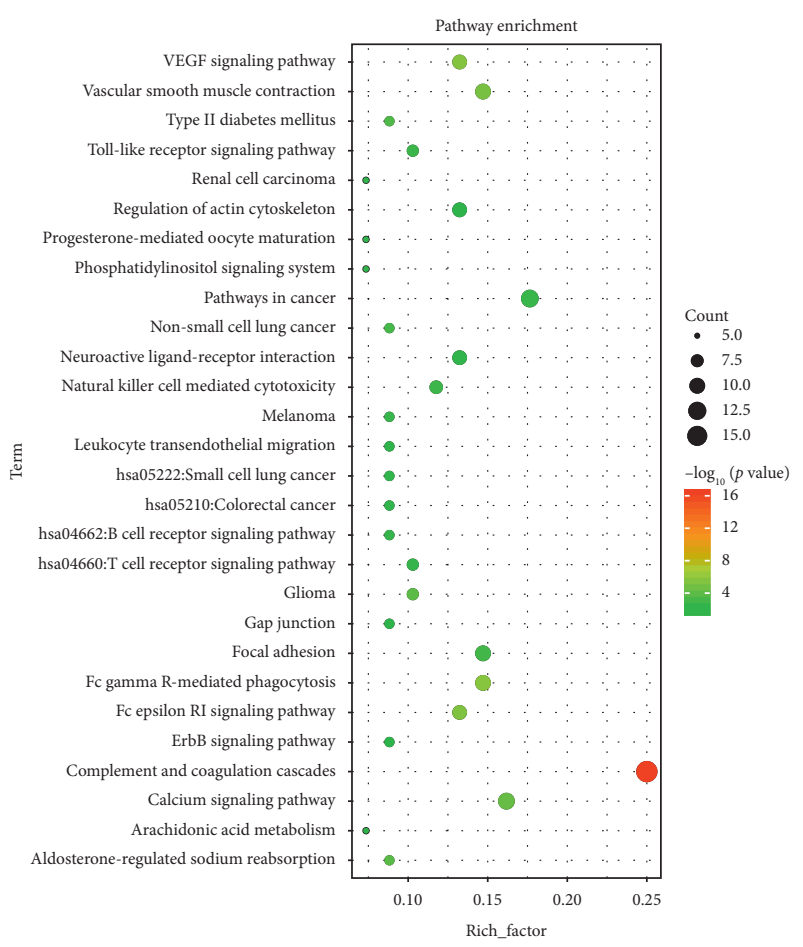

(d)

Figure 6: Gene ontology enrichment and KEGG pathway analysis of the targets of QXH related to coagulopathy module. (a) Biological process. (b) Molecular function. (c) Cellular component. (d) KEGG Pathway.

atherosclerosis in the vessel wall. But the PPAR $\gamma$ expression in monocytes increased the expression of CD36, leading to an enhanced macrophage uptake of oxidized LDL, thus enhancing foam cell formation [68].

In the coagulopathy network module, 68 targets participated in signaling pathways such as complement and coagulation cascades, VEGF signaling pathway, vascular smooth muscle contraction, calcium signaling pathway, arachidonic acid metabolism, and long-term depression. F2, F3, F7, F9, F10, SERPINE1, and SERPIND1 were included by complement and coagulation cascades, which acted on blood coagulation, platelet activation, blood homeostasis, inflammation, and wound healing. Studies have shown that APTT and PT reflect changes in the coagulation factor of the endogenous and exogenous coagulation pathways, respectively. TT and FIB are related to the common coagulation pathway in plasma [36]. However, the analysis results of coagulopathy network module fully support the rationality of using APTT, TT, PT, and FIB to evaluate QXH on improving coagulation function in QS-BSS. Another study on identifying the compounds of DS on intervening thrombotic diseases revealed that tanshinone IIB could improvemicrocirculation and prevent thrombosis involved in complement and coagulation cascades based on UPLCLTQ-Orbitrap MS [69], which is also comparable to our findings.

Analysis of the targets overlapped such as PTGS1, PTGS2, PIK3CG, and PECAM1 in the pathological processes of hemorheological abnormality and coagulopathy implied that inflammation and immunity were also important for QS-BSS which need further study. Complement and coagulation cascades, arachidonic acid metabolism, and VEGF signaling pathway were shared in hemorheological abnormality and coagulopathy, which need more attention, and further experimental validation will be carried out.

However, although we have predicted the main active compounds of QXH through network pharmacology and some of which has been verified by UPLC-Q/TOF-MS, to clarify the substance basis of the pharmacodynamic effect of QXH, further study on the migrating components absorbed into blood by serum pharmacochemistry based on animal experiments is needed [70]. Meanwhile, the network analysis 


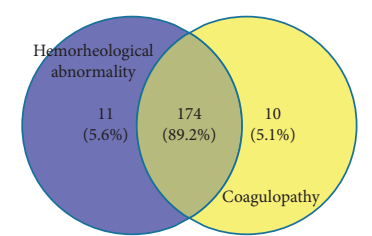

(a)

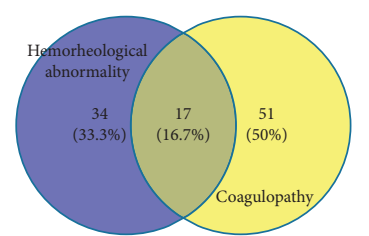

(b)

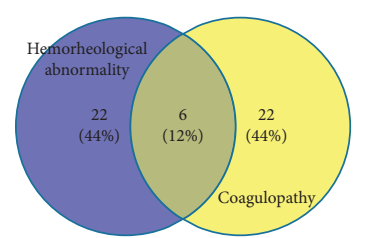

(c)

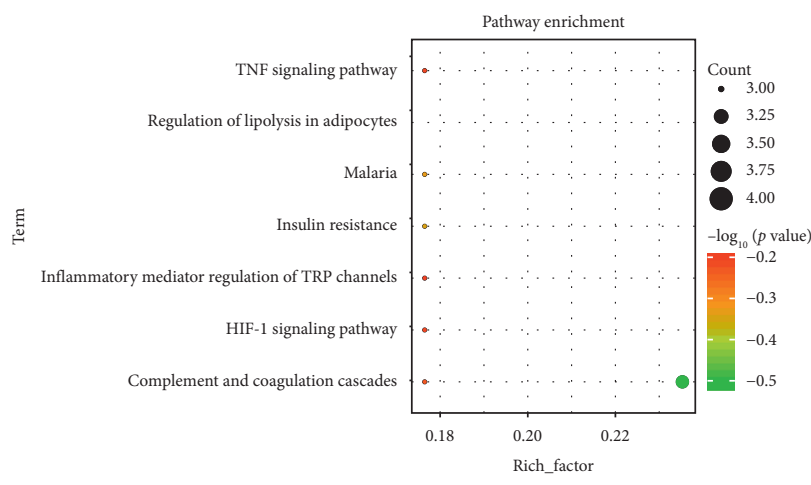

(d)

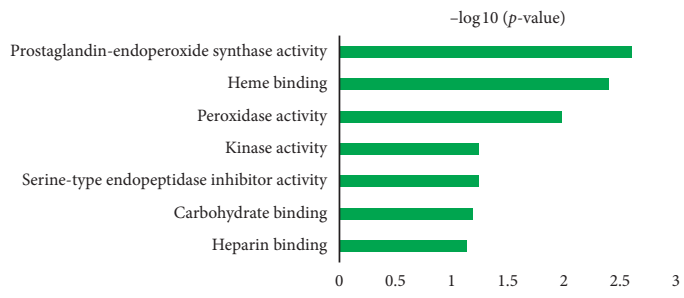

(f)

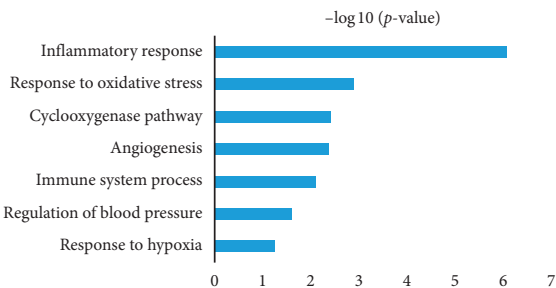

(e)

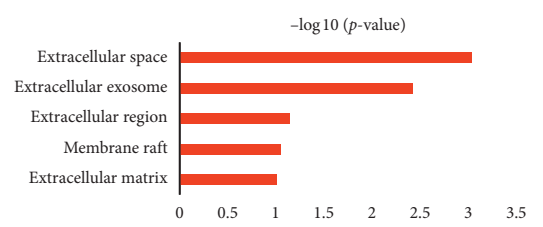

(g)

FIGURE 7: The analysis of overlapped compounds, targets, and pathways of the pathological processes: hemorheological abnormality and coagulopathy. (a) The overlapped compounds. (b) The overlapped targets. (c) The overlapped pathways. (d) KEGG pathway of the overlapped targets. (e) Biological process. (f) Molecular function. (g) Cellular component.

also provided a vital reference for experimental investigations of hub targets and signaling pathways of QXH in treating QS-BSS.

\section{Conclusions}

Through the pharmacodynamic evaluation of QXH based on animal experiments and the underlying pharmacological mechanism prediction of QXH, our study has proved that the effect of QXH in the treatment of QS-BSS is significant, and the underlying pharmacological mechanisms might be related to complement and coagulation cascades, leukocyte transendothelial migration, PPAR signaling pathway, VEGF signaling pathway, arachidonic acid metabolism, inflammatory mediator regulation of TRP channels, and TNF signaling pathway, for which the further experimental validation is essential.

\section{Abbreviations}

QXH: $\quad$ Qixuehe capsule

QXH-L: $\quad$ Low-dose QXH-intervened

QXH-M: Middle-dose QXH-intervened

QXH-H: High-dose QXH-intervened

TCM: Traditional Chinese medicine
Adr: $\quad$ Adrenaline hydrochloride

CDDP: $\quad$ Compound danshen dripping pills

PT: $\quad$ Prothrombin time

APTT: $\quad$ Activated partial thromboplastin time

TT: $\quad$ Thrombin time

FIB: $\quad$ Fibrinogen content

WBV: Whole blood viscosity

PV: $\quad$ Plasma viscosity

PCA: $\quad$ Principal component analysis

PLS: $\quad$ Partial least squares

CV- Analysis of variance testing of cross-validated

ANOVA: predictive residuals

UPLC: Ultra-performance liquid chromatography

MS: $\quad$ Mass spectrometer

TCMSP: Traditional Chinese medicine systems pharmacology database and analysis platform

TTD: $\quad$ Therapeutic targets database

CTD: $\quad$ Comparative toxicogenomics database

SEA: $\quad$ Similarity ensemble approach

OMIM: $\quad$ Online Mendelian inheritance in man database

OB: $\quad$ Oral bioavailability

Caco-2: Caco-cell permeability

DL: $\quad$ Drug-likeness

KEGG: Kyoto Encyclopedia of Genes and Genomes 


$\begin{array}{ll}\text { DAVID: } & \begin{array}{l}\text { Database for Annotation, Visualization and } \\ \text { Integrated Discovery }\end{array} \\ \text { DG: } & \text { Angelicae Sinensis Radix } \\ \text { CS: } & \text { Paeoniae Radix Rubra } \\ \text { CX: } & \text { Chuanxiong Rhizoma } \\ \text { TR: } & \text { Persicae Semen } \\ \text { HH: } & \text { Carthami Flos } \\ \text { CH: } & \text { Bupleuri Radix } \\ \text { XF: } & \text { Cyperi Rhizoma } \\ \text { DS: } & \text { Salviae Miltiorrhizae Radix et Rhizoma } \\ \text { YHS: } & \text { Corydalis Rhizoma } \\ \text { JG: } & \text { Platycodonis Radix } \\ \text { ZQ: } & \text { Aurantii Fructus } \\ \text { WY: } & \text { Linderae Radix } \\ \text { NX: } & \text { Achyranthis Bidentatae Radix } \\ \text { SM: } & \text { Cimicifugae Rhizoma } \\ \text { GC: } & \text { Glycyrrhizae Radix et Rhizoma. }\end{array}$

\section{Data Availability}

The data that support the findings of this study are available in the Supplementary Materials and openly available in the PubChem (https://pubchem.ncbi.nlm.nih.gov), ChemSpider (http://www.chemspider.com), TCMSP (http://lsp. nwu.edu.cn/index.php), TCM-MESH (http://mesh.tcm. microbioinformatics.org), STITCH (http://stitch.embl.de/), Therapeutic Targets Database (TTD, http://bidd.nus.edu.sg/ group/ttd/), Comparative Toxicogenomics Database (CTD, http://ctdbase.org/), the Similarity Ensemble Approach (SEA, http://sea.bkslab.org/), Swiss Target Prediction webserver (http://www.swisstargetprediction.ch), Online Mendelian Inheritance in Man Database (OMIM, http://www. omim.org/), and Uniprot (https://www.uniprot.org).

\section{Conflicts of Interest}

The authors declare that they have no conflicts of interest.

\section{Authors' Contributions}

Yu-Xi Huang and Ding-Qiao Xu contribute equally to this article.

\section{Acknowledgments}

This study was supported by grants from Key Research and Development Program of Shaanxi (2019ZDLSF04-05), National Key R\&D Program of China (2019YFC1711000), the National Natural Science Foundation of China (81773882, 81974522, and 81903786), and the Natural Science Foundation of Shaanxi Province (2019JQ-054). This research was also financially supported by the Subject Innovation Team of Shaanxi University of Chinese Medicine (2019-YL10).

\section{Supplementary Materials}

Table S1: the OB, DL, and Caco-2 of the active compounds in QXH. Table S2: the active compounds involved in hemorheological abnormality module and coagulopathy module.
Table S3: the candidate targets involved in hemorheological abnormality module and coagulopathy module. Table S4: the overlapped targets in hemorheological abnormality module and coagulopathy module. Figure S1: PCA score plots of hemorheology and coagulation function indexes among all groups. Figure S2: the permutation tests $(n=200)(\mathrm{A})$ and CV-AVNOA tests (B) for the PLS-DA model among all groups. Figure S3: PLS-DA score plots of hemorheology and coagulation function indexes between control and model groups. Figure S4: the permutation tests $(n=200)(\mathrm{A})$ and CV-AVNOA tests (B) for the PLS-DA model between control and model groups. Figure S5: base peak intensity chromatograms of 25 standards in positive ion mode or negative ion mode. Figure S6: base peak intensity chromatograms of QXH methanol extracts (A) in positive ion mode and $(C)$ in negative ion mode; base peak intensity chromatograms of QXH water extracts (B) in positive ion mode and (D) in negative ion mode by UPLC-Q/TOF-MS. Figure S7: the HH-CH-TH network of QXH (hemorheological abnormality module; red node: herbs, green node: compounds, and blue node: targets) (the original picture for Figure 3). Figure S8: the HC-CC-TC network of QXH (coagulopathy module, red node: herbs, green node: compounds, and blue node: targets) (the original picture for Figure 5). (Supplementary Materials)

\section{References}

[1] A. S. Peña, D. A. Doherty, H. C. Atkinson, M. Hickey, R. J. Norman, and R. Hart, "The majority of irregular menstrual cycles in adolescence are ovulatory: results of a prospective study," Archives of Disease in Childhood, vol. 103, no. 3, pp. 235-239, 2018.

[2] Y. Zhang, X. Mao, J. Su et al., "A network pharmacologybased strategy deciphers the underlying molecular mechanisms of qixuehe capsule in the treatment of menstrual disorders," Chinese Medicine, vol. 12, no. 1, p. 23, 2017.

[3] G. Chen, J. Gao, H. He et al., "Identification of differentially expressed non-coding RNAs and mRNAs involved in qi stagnation and blood stasis syndrome," Experimental and Therapeutic Medicine, vol. 17, no. 2, pp. 1206-1223, 2018.

[4] H. He, G. Chen, J. Gao et al., "Xue-Fu-Zhu-Yu capsule in the treatment of qi stagnation and blood stasis syndrome: a study protocol for a randomised controlled pilot and feasibility trial," Trials, vol. 19, no. 1, p. 515, 2018.

[5] C. Zhang, C. Huang, M. Wang, X. Kong, G. Liu, and J. Wang, "Correlation analysis between traditional Chinese medicine syndromes and gastrointestinal bleeding after percutaneous coronary intervention," Evidence-Based Complementary and Alternative Medicine, vol. 2018, Article ID 7356546, 10 pages, 2018.

[6] S.-M. Li, H. Xu, and K.-J. Chen, "The diagnostic criteria of blood-stasis syndrome: considerations for standardization of pattern identification," Chinese Journal of Integrative Medicine, vol. 20, no. 7, pp. 483-489, 2014.

[7] J. Liao, Y. Liu, and J. Wang, "Identification of more objective biomarkers for blood-stasis syndrome diagnosis," BMC Complementary and Alternative Medicine, vol. 16, no. 1, p. 371, 2016.

[8] B. Park, S. You, J. Jung, J. A. Lee, K. J. Yun, and M. S. Lee, "Korean studies on blood stasis: an overview," Evidence-Based 
Complementary and Alternative Medicine, vol. 2015, Article ID 316872, 7 pages, 2015.

[9] L. Ma, X. Zhang, X. Xu et al., "Compatibility principle in the Tanyu Tongzhi formula revealed by a cell-based analysis," Journal of Ethnopharmacology, vol. 231, pp. 507-515, 2019.

[10] Z. Xing, Z. Xia, W. Peng et al., "Xuefu Zhuyu decoction, a traditional Chinese medicine, provides neuroprotection in a rat model of traumatic brain injury via an anti-inflammatory pathway," Scientific Reports, vol. 6, no. 1, Article ID 20040, 2016.

[11] R. Q. Tan, Z. Zhang, J. Ju, and J. H. Ling, "Effect of Chaihu Shugan powder-contained serum on glutamate-induced autophagy of interstitial cells of Cajal in the rat gastric antrum," Evidence-Based Complementary and Alternative Medicine, vol. 2019, Article ID 7318616, 7 pages, 2019.

[12] N. Wang, P. Xu, X. Wang et al., "Integrated pathological cell fishing and network pharmacology approach to investigate main active components of Er-Xian decotion for treating osteoporosis," Journal of Ethnopharmacology, vol. 241, Article ID 111977, 2019.

[13] H. Zuo, Q. Zhang, S. Su, Q. Chen, F. Yang, and Y. Hu, “A network pharmacology-based approach to analyse potential targets of traditional herbal formulas: an example of Yu Ping Feng decoction," Scientific Reports, vol. 8, no. 1, Article ID 11418, 2018

[14] Y. Zhong, J. Luo, T. Tang et al., "Exploring pharmacological mechanisms of Xuefu Zhuyu decoction in the treatment of traumatic brain injury via a network pharmacology approach," Evidence-Based Complementary and Alternative Medicine, vol. 2018, Article ID 8916938, 20 pages, 2018.

[15] X. Li, X. Xu, J. Wang et al., "A system-level investigation into the mechanisms of Chinese traditional medicine: compound Danshen formula for cardiovascular disease treatment," PLoS One, vol. 7, no. 9, Article ID e43918, 2012.

[16] Q. Ma, P. L. Li, Y. L. Hua et al., "Effects of Tao-Hong-Si-Wu decoction on acute blood stasis in rats based on a LC-Q/TOFMS metabolomics and network approach," Biomedical Chromatography, vol. 32, no. 4, Article ID e4144, 2017.

[17] P. Liu, E.-X. Shang, Y. Zhu, J.-G. Yu, D.-W. Qian, and J.-A. Duan, "Comparative analysis of compatibility effects on invigorating blood circulation for cyperi rhizoma series of herb pairs using untargeted metabolomics," Frontiers in Pharmacology, vol. 8 , no. 677, 2017.

[18] W. Li, J. A. Duan, P. Liu et al., "Study on evaluation system for gynecological disease model characterized by qi stagnation and blood stasis syndrome with female SD rats," China Journal of Chinese Materia Medica, vol. 38, no. 8, pp. 12111218, 2013.

[19] J. Ru, P. Li, J. Wang et al., "TCMSP: a database of systems pharmacology for drug discovery from herbal medicines," Journal of Cheminformatics, vol. 6, no. 1, p. 13, 2014.

[20] S. J. Yue, L. T. Xin, Y. C. Fan et al., "Herb pair DangguiHonghua: mechanisms underlying blood stasis syndrome by system pharmacology approach," Scientific Reports, vol. 7, no. 1, Article ID 40318, 2017.

[21] X.-Q. Shi, S.-J. Yue, Y.-P. Tang et al., "A network pharmacology approach to investigate the blood enriching mechanism of Danggui buxue decoction," Journal of Ethnopharmacology, vol. 235, pp. 227-242, 2019.

[22] X. Xu, W. Zhang, C. Huang et al., "A novel chemometric method for the prediction of human oral bioavailability," International Journal of Molecular Sciences, vol. 13, no. 6, pp. 6964-6982, 2012.
[23] Y. Wan, L. Xu, Z. Liu et al., "Utilising network pharmacology to explore the underlying mechanism of Wumei Pill in treating pancreatic neoplasms," BMC Complementary and Alternative Medicine, vol. 19, no. 1, p. 158, 2019.

[24] C. Wang, Q. Ren, X. T. Chen et al., "System pharmacologybased strategy to decode the synergistic mechanism of Zhi-Zhu Wan for functional dyspepsia," Frontiers in Pharmacology, vol. 9, p. 841, 2018.

[25] E. E. Bolton, Y. Wang, P. A. Thiessen, and S. H. Bryant, "PubChem: integrated platform of small molecules and biological activities," Annual Reports in Computational Chemistry, vol. 4, pp. 217-241, 2008.

[26] H. E. Pence and A. Williams, "ChemSpider: an online chemical information resource," Journal of Chemical Education, vol. 87, no. 11, pp. 1123-1124, 2010.

[27] S. J. Yue, J. Liu, W. W. Feng et al., "System pharmacologybased dissection of the synergistic mechanism of Huangqi and Huanglian for diabetes mellitus," Frontiers in Pharmacology, vol. 8, p. 694, 2017.

[28] M. Kuhn, C. von Mering, M. Campillos, L. J. Jensen, and P. Bork, "STITCH: interaction networks of chemicals and proteins," Nucleic Acids Research, vol. 36, no. 1, pp. D684D688, 2007.

[29] F. Zhu, Z. Shi, C. Qin et al., "Therapeutic target database update 2012: a resource for facilitating target-oriented drug discovery," Nucleic Acids Research, vol. 40, no. D1, pp. D1128-D1136, 2012.

[30] A. P. Davis, C. J. Grondin, R. J. Johnson et al., "The comparative toxicogenomics database: update 2017," Nucleic Acids Research, vol. 45, no. D1, pp. D972-D978, 2017.

[31] M. J. Keiser, B. L. Roth, B. N. Armbruster, P. Ernsberger, J. J. Irwin, and B. K. Shoichet, "Relating protein pharmacology by ligand chemistry," Nature Biotechnology, vol. 25, no. 2, pp. 197-206, 2007.

[32] D. Gfeller, A. Grosdidier, M. Wirth, A. Daina, O. Michielin, and V. Zoete, "SwissTargetPrediction: a web server for target prediction of bioactive small molecules," Nucleic Acids Research, vol. 42, no. W1, pp. W32-W38, 2014.

[33] A. Hamosh, A. F. Scott, J. S. Amberger, C. A. Bocchini, and V. A. McKusick, "Online Mendelian inheritance in man (OMIM), a knowledgebase of human genes and genetic disorders," Nucleic Acids Research, vol. 33, no. 1, pp. D514D517, 2005.

[34] The UniProt Consortium, "UniProt: the universal protein knowledgebase," Nucleic Acids Research, vol. 45, no. D1, pp. D158-D169, 2017.

[35] G. Dennis, B. T. Sherman, D. A. Hosack et al., "DAVID: database for annotation, visualization, and integrated discovery," Genome Biology, vol. 4, no. 9, p. R60, 2003.

[36] M. E. Smoot, K. Ono, J. Ruscheinski, P.-L. Wang, and T. Ideker, "Cytoscape 2.8: new features for data integration and network visualization," Bioinformatics, vol. 27, no. 3, pp. 431-432, 2011.

[37] X. Zhang, P. Li, Y. Hua et al., "Urinary metabolomics study the mechanism of Taohong Siwu decoction intervention in acute blood stasis model rats based on liquid chromatography coupled to quadrupole time-of-flight mass spectrometry," Journal of Chromatography B, vol. 1074-1075, pp. 51-60, 2018.

[38] S. Sun, F. Gong, P. Liu, and Q. Miao, "Metformin combined with quercetin synergistically repressed prostate cancer cells via inhibition of VEGF/PI3K/Akt signaling pathway," Gene, vol. 664, pp. 50-57, 2018.

[39] R. E. Roberts, S. Allen, A. P. Y. Chang et al., "Distinct mechanisms of relaxation to bioactive components from 
chamomile species in porcine isolated blood vessels," Toxicology and Applied Pharmacology, vol. 272, no. 3, pp. 797-805, 2013.

[40] K. W. Makar, E. M. Poole, A. J. Resler et al., "COX-1 (PTGS1) and COX-2 (PTGS2) polymorphisms, NSAID interactions, and risk of colon and rectal cancers in two independent populations," Cancer Causes \& Control, vol. 24, no. 12, pp. 2059-2075, 2013.

[41] D. H. A. Yang, C.-F. Hsu, C.-Y. Lin, J.-Y. Guo, W. C. Y. Yu, and V. H. S. Chang, "Krüppel-like factor 10 upregulates the expression of cyclooxygenase 1 and further modulates angiogenesis in endothelial cell and platelet aggregation in genedeficient mice," The International Journal of Biochemistry \& Cell Biology, vol. 45, no. 2, pp. 419-428, 2013.

[42] J. Hellmann, Y. Tang, M. J. Zhang et al., "Atf3 negatively regulates Ptgs2/Cox2 expression during acute inflammation," Prostaglandins \& Other Lipid Mediators, vol. 116-117, pp. 49-56, 2015.

[43] H. H. Gao, L. B. Gao, and J. M. Wen, "Genetic polymorphisms in the ESR1 gene and cerebral infarction risk: a meta-analysis," DNA and Cell Biology, vol. 33, no. 9, pp. 605-615, 2014.

[44] J. C. Chapin and K. A. Hajjar, "Fibrinolysis and the control of blood coagulation," Blood Reviews, vol. 29, no. 1, pp. 17-24, 2015.

[45] C. Demacq, V. B. Vasconcellos, T. C. Izidoro-Toledo et al., "Vascular endothelial growth factor (VEGF) and endothelial nitric oxide synthase (NOS3) polymorphisms are associated with high relapse risk in childhood acute lymphoblastic leukemia (ALL)," Clinica Chimica Acta, vol. 411, no. 17-18, pp. 1335-1340, 2010.

[46] M. Zhao, Y. Chen, C. Wang et al., "Systems pharmacology dissection of multi-scale mechanisms of action of Huo-XiangZheng-Qi formula for the treatment of gastrointestinal diseases," Frontiers in Pharmacology, vol. 9, p. 1448, 2019.

[47] M. Wang, F. L. Xiao, Y. J. Mao, L. L. Ying, B. Zhou, and Y. Li, "Quercetin decreases the triglyceride content through the PPAR signalling pathway in primary hepatocytes of broiler chickens," Biotechnology \& Biotechnological Equipment, vol. 33, no. 1, pp. 1000-1010, 2019.

[48] S. Somacal, C. G. Figueiredo, A. Quatrin et al., "The antiatherogenic effect of bixin in hypercholesterolemic rabbits is associated to the improvement of lipid profile and to its antioxidant and anti-inflammatory effects," Molecular and Cellular Biochemistry, vol. 403, no. 1-2, pp. 243-253, 2015.

[49] J. Che, B. Liang, Y. Zhang, Y. Wang, J. Tang, and G. Shi, "Kaempferol alleviates ox-LDL-induced apoptosis by upregulation of autophagy via inhibiting PI3K/Akt/mTOR pathway in human endothelial cells," Cardiovascular $\mathrm{Pa}$ thology, vol. 31, pp. 57-62, 2017.

[50] L. Chapado, P. J. Linares-Palomino, S. Salido, J. Altarejos, J. A. Rosado, and G. M. Salido, "Synthesis and evaluation of the platelet antiaggregant properties of phenolic antioxidants structurally related to rosmarinic acid," Bioorganic Chemistry, vol. 38, no. 3, pp. 108-114, 2010.

[51] Y. Wang, X. Yi, K. Ghanam, S. Zhang, T. Zhao, and X. Zhu, "Berberine decreases cholesterol levels in rats through multiple mechanisms, including inhibition of cholesterol absorption," Metabolism, vol. 63, no. 9, pp. 1167-1177, 2014.

[52] W. Kong, J. Wei, P. Abidi et al., "Berberine is a novel cholesterol-lowering drug working through a unique mechanism distinct from statins," Nature Medicine, vol. 10, no. 12, pp. 1344-1351, 2004.

[53] Y. Wang, Y. Han, F. Chai et al., "The antihypercholesterolemic effect of columbamine from rhizoma coptidis in HFHC-diet induced hamsters through HNF-4 $\alpha /$ FTF-mediated CYP7A1 activation," Fitoterapia, vol. 115, pp. 111-121, 2016.

[54] Y.-x. Fei, S.-q. Wang, L.-j. Yang et al., "Salvia miltiorrhiza Bunge (Danshen) extract attenuates permanent cerebral ischemia through inhibiting platelet activation in rats," Journal of Ethnopharmacology, vol. 207, pp. 57-66, 2017.

[55] G. Lagaly and S. Ziesmer, "Colloid chemistry of clay minerals: the coagulation of montmorillonite dispersions," Advances in Colloid and Interface Science, vol. 100-102, pp. 105-128, 2003.

[56] X. Li, L. L. Mazaleuskaya, L. L. Ballantyne, H. Meng, G. A. FitzGerald, and C. D. Funk, "Genomic and lipidomic analyses differentiate the compensatory roles of two COX isoforms during systemic inflammation in mice," Journal of Lipid Research, vol. 59, no. 1, pp. 102-112, 2017.

[57] E. Gavriilaki and R. A. Brodsky, "25-complement-mediated coagulation disorders: paroxysmal nocturnal hemoglobinuria and atypical hemolytic uremic syndrome," in Consultative Hemostasis and Thrombosis, pp. 473-490, Elsevier, Amsterdam, Netherlands, 4th edition, 2019.

[58] X. Liu, L. Hao, S. Zhang et al., "Genetic repression of mouse VEGF expression regulates coagulation cascade," IUBMB Life, vol. 62, no. 11, pp. 819-824, 2010.

[59] Y. Liu, H. J. Yin, D. Z. Shi, and K. J. Chen, "Chinese herb and formulas for promoting blood circulation and removing blood stasis and antiplatelet therapies," Evidence-Based Complementary and Alternative Medicine, vol. 2012, Article ID 184503, 8 pages, 2012.

[60] S. Zorgo, G. Purebl, and A. Zana, "A qualitative study of culturally embedded factors in complementary and alternative medicine use," BMC Complementary and Alternative Medicine, vol. 18, no. 1, p. 25, 2018.

[61] Y. Dong, P. Qiu, R. Zhu et al., "A combined phytochemistry and network pharmacology approach to reveal the potential antitumor effective substances and mechanism of Phellinus igniarius," Frontiers in Pharmacology, vol. 10 , no. 266, 2019.

[62] C. Lv, C. Liu, Z. Yao et al., "The clinical pharmacokinetics and pharmacodynamics of warfarin when combined with compound danshen: a case study for combined treatment of coronary heart diseases with atrial fibrillation," Frontiers in Pharmacology, vol. 8, p. 826, 2017.

[63] J. Cao, Z. Chen, Y. Zhu et al., "Huangqi-Honghua combination and its main components ameliorate cerebral infarction with Qi deficiency and blood stasis syndrome by antioxidant action in rats," Journal of Ethnopharmacology, vol. 155, no. 2, pp. 1053-1060, 2014.

[64] D. Shen, N. Ma, Y. Yang et al., "UPLC-Q-TOF/MS-based plasma metabolomics to evaluate the effects of aspirin eugenol ester on blood stasis in rats," Molecules, vol. 24, no. 13, Article ID 2380, 2019.

[65] J. Zhou, Z. Song, M. Han et al., "Evaluation of the antithrombotic activity of Zhi-Xiong capsules, a traditional Chinese medicinal formula, via the pathway of anti-coagulation, antiplatelet activation and anti-fibrinolysis," Biomedicine \& Pharmacotherapy, vol. 97, pp. 1622-1631, 2018.

[66] G. M. Annich, "Extracorporeal life support: the precarious balance of hemostasis," Journal of Thrombosis and Haemostasis, vol. 13, no. S1, pp. S336-S342, 2015.

[67] J. Feng, J. Han, S. F. Pearce et al., "Induction of CD36 expression by oxidized LDL and IL- 4 by a common signaling pathway dependent on protein kinase C and PPAR-gamma," Journal of Lipid Research, vol. 41, no. 5, pp. 688-696, 2000.

[68] Q. Jia, H. Cao, D. Shen et al., "Quercetin protects against atherosclerosis by regulating the expression of PCSK9, CD36, 
$\operatorname{PPAR} \gamma, \mathrm{LXR} \alpha$ and ABCA1," International Journal of Molecular Medicine, vol. 44, no. 3, pp. 893-902, 2019.

[69] P. Ge, L. Wen, X. Wang, J. Zhang, and G. Xu, "Rapidly identify compounds from Danshen by using ultra-high-performance liquid chromatography coupled with linear ion trap-orbitrap mass spectrometer and predict its mechanisms of intervening thrombotic diseases," Journal of Liquid Chromatography \& Related Technologies, vol. 42, no. 5-6, pp. 115-121, 2019.

[70] A.-H. Zhang, H. Sun, G.-L. Yan, Y. Han, Q.-Q. Zhao, and X.-J. Wang, "Chinmedomics: a powerful approach integrating metabolomics with serum pharmacochemistry to evaluate the efficacy of traditional Chinese medicine," Engineering, vol. 5, no. 1 , pp. 60-68, 2019. 